\title{
Formation Mechanism of Anisotropic RDX-TNT Core-Shell Nanoparticles and their Influence onto Nanodiamond Detonation Syntheses
}

Jakob Hübner, Vincent Pichot, Emeline Lobry, Tanja Deckert-Gaudig, Volker Deckert, Denis Spitzer

Submitted date: 19/03/2020 Posted date: 19/03/2020

Licence: CC BY-NC-ND 4.0

Citation information: Hübner, Jakob; Pichot, Vincent; Lobry, Emeline; Deckert-Gaudig, Tanja; Deckert, Volker; Spitzer, Denis (2020): Formation Mechanism of Anisotropic RDX-TNT Core-Shell Nanoparticles and their Influence onto Nanodiamond Detonation Syntheses. ChemRxiv. Preprint.

https://doi.org/10.26434/chemrxiv.12005625.v1

Mixtures of RDX and TNT or hexolite mixtures are well known precursors for nanodiamond detonation syntheses. In this study diverse nanoscale hexolite mixtures varying in mass ratios of RDX and TNT are synthesized via Spray Flash Evaporation. The hexolite mixtures are characterized by confocal Raman spectroscopy and tip-enhanced Raman spectroscopy (TERS) to obtain information about their molecular structure composition. The marker bands of pristine RDX and TNT enable the identification and distinction of both compounds in the Raman spectra. Confocal Raman spectroscopy indicates an intermixture of RDX and TNT molecules on the nanoscale since both marker bands are detected in all spectra. TERS investigations of single hexolite particles reveal that the particle surfaces are mainly composed of TNT. The comparison of confocal Raman and TERS results suggests that (depending on the mass ratio) hexolite particles are either inhomogeneous patchy RDX/TNT nanoparticles or anisotropic RDX/TNT core-shell nanoparticles. A building mechanism to explain the formation/growth of those nanoparticles is derived from the spectroscopic data and the dynamics of the SFE process. Finally, a correlation between the TNT shell thickness, the symmetry of the anisotropic hexolite precursor nanoparticles, and the resulting nanodiamond sizes is discussed in detail.

File list (2)

Formation Mechanism of Anisotropic RDXTNT CoreShell ... (2.51 MiB) view on ChemRxiv • download file Supporting Informnation - Formation Mechanism of Anis... (795.94 KiB) view on ChemRxiv • download file 


\section{Formation Mechanism of Anisotropic RDX/TNT Core/Shell Nanoparticles and their Influence onto Nanodiamond Detonation Syntheses}

Jakob Hübner ${ }^{[\mathrm{a}]}$, Vincent Pichot ${ }^{[\mathrm{a}]}$, Emeline Lobry ${ }^{[\mathrm{a}]}$, Tanja Deckert-Gaudig ${ }^{[\mathrm{b}]}$, Volker Deckert $^{[\mathrm{b}, \mathrm{c}, \mathrm{d}]}$, Denis Spitzer ${ }^{[\mathrm{a}]}$

[a] Nanomatériaux pour les Systèmes Sous Sollicitations Extrêmes (NS3E), ISL-CNRSUNISTRA UMR 3208, French-German Research Institute of Saint-Louis, 5, rue du Général Cassagnou, B.P. 70034, 68301 Saint-Louis, France

[b] Leibniz Institute of Photonic Technology (IPHT), Albert-Einstein-Str. 9, 07745 Jena, Germany

[c] Institute of Physical Chemistry and Abbe Center of Photonics, Friedrich-Schiller-University Jena, Helmholtzweg 4, 07743 Jena, Germany

[d] Institute of Quantum Science and Engineering, Texas A\&M University, College Station, TX 77843-4242, USA

\section{Corresponding Author}

Correspondence should be addressed to jakob.huebner@isl.eu

\section{Keywords}

Hexolite mixtures, TERS, anisotropic core-shell nanoparticles, building mechanism, nanodiamond formation 


\begin{abstract}
Mixtures of RDX and TNT or hexolite mixtures are well known precursors for nanodiamond detonation syntheses. In this study diverse nanoscale hexolite mixtures varying in mass ratios of RDX and TNT are synthesized via Spray Flash Evaporation. The hexolite mixtures are characterized by confocal Raman spectroscopy and tip-enhanced Raman spectroscopy (TERS) to obtain information about their molecular structure composition. The marker bands of pristine RDX and TNT enable the identification and distinction of both compounds in the Raman spectra. Confocal Raman spectroscopy indicates an intermixture of RDX and TNT molecules on the nanoscale since both marker bands are detected in all spectra. TERS investigations of single hexolite particles reveal that the particle surfaces are mainly composed of TNT. The comparison of confocal Raman and TERS results suggests that (depending on the mass ratio) hexolite particles are either inhomogeneous patchy RDX/TNT nanoparticles or anisotropic RDX/TNT core-shell nanoparticles. A building mechanism to explain the formation/growth of those nanoparticles is derived from the spectroscopic data and the dynamics of the SFE process. Finally, a correlation between the TNT shell thickness, the symmetry of the anisotropic hexolite precursor nanoparticles, and the resulting nanodiamond sizes is discussed in detail.
\end{abstract}




\section{Introduction}

Since the first detonation synthesis of nanodiamonds in the USSR back in the 1960s, nanodiamonds have become a focal point of interest in various fields of science and industries. ${ }^{1-}$ ${ }^{2}$ Functionalized nanodiamonds found their use inter alia in biomedical approaches as non-toxic compounds for biomedical imaging or as drug and gene delivery agents. ${ }^{3-8}$ In addition to the medical approaches, nanodiamonds are used in electronic, chemical, material and energetic applications. ${ }^{9-13}$ Probably due to the broad field of application and the constantly growing demand for nanodiamonds, research increasingly aims at gaining insights into the mechanism of nanodiamond formation in detonation syntheses. ${ }^{14-22}$ A detailed knowledge of the building mechanism is crucial for the adaption of nanodiamond sizes and size distribution associated with their application. Furthermore, especially the structure of the precursor material influences strongly the resulting nanodiamonds. Pichot et al. demonstrated that nanoscale RDX and TNT (hexolite) precursor particles result in the formation of nanodiamonds characterized by smaller mean sizes and narrower size distribution as obtained by employing micron sized precursor materials. ${ }^{23-24}$ Using nanostructured RDX and TNT precursor particles, they obtained nanodiamonds defined by particle mean sizes between $2.8 \mathrm{~nm}-4.2 \mathrm{~nm}$ depending on the precursor compositions. Since the precursor structure affects strongly the resulting nanodiamonds different nanoscale hexolite mixtures of various RDX and TNT mass ratios are investigated in this study. All presented nano hexolites were produced by Spray Flash Evaporation (SFE). This technique allows the continuous production of organic submicron- and nanoparticles. ${ }^{25-26}$ During the SFE process a preheated and pressurized solution containing one (or more) different components is sprayed through a hollow cone nozzle into a permanently evacuated reaction chamber. The strong pressure and temperature difference in front and behind the nozzle implement an immediate evaporation of the dissolved micro droplets resulting in the release of submicron or nanoparticles. Depending on the composition of the dissolved precursors a huge variety of submicron or nanoscale particles can be obtained. ${ }^{23-24,26-32}$

However, the characterization of SFE-produced hexolite composites turned out to be quite difficult using common measurement methods for several reasons. On the one hand, electron microscopy and energy dispersive X-ray spectroscopy failed to determine the structure and composition of single hexolite particles although these techniques allow structure analysis of other energetic materials. ${ }^{33}$ Due to the thermal instability of RDX and TNT, hexolite, nanoparticles tend to dissociate under the electron beam. Other standard methods like infrared and far-field Raman spectroscopy or X-ray diffraction may provide molecular and crystallographic information but due to the lack of an adequate sensitivity and resolution, the 
characterization of single nanoparticles remain impossible. Furthermore, X-ray photoelectron spectroscopy (XPS) failed to investigate structures and chemical compositions of SFEproduced hexolite mixtures since nanostructured TNT sublimates rapidly under the high vacuum conditions of XPS. Thus, AFM-based tip-enhanced Raman spectroscopy (TERS) was chosen for hexolite nanoparticle characterization since it provides ultrahigh sensitivity and spatial resolution at a low energetic load on the sample (compared to electron microscopy methods). In AFM-TERS a common cantilever tip is coated with silver (or gold) nanoparticle and irradiated with a laser wavelength that matches the plasmon resonance of the nanoparticles. The incident laser light causes a collective oscillation of the conductive electrons within the silver nanoparticle. This so called localized surface plasmon resonance (LSPR) leads to an enhancement of the electrical field close to the silver particle surface, which rapidly decays within a few nanometers. ${ }^{34-37}$ The strong field enhancement enables spectroscopic investigations of nanoparticles, molecular monolayers and even single molecules. ${ }^{32-33}$, 38-44 Furthermore, Deckert-Gaudig et al. successfully analyzed hexolite RDX/TNT core-shell nanoparticles with TERS. ${ }^{44}$ In this contribution, the synthesis and characterization of diverse SFE produced anisotropic RDX/TNT core-shell nanoparticles and inhomogeneous RDX/TNT nanoparticles are presented. Furthermore, a building mechanism of these nanoparticles including the spectroscopic data and the dynamics of the SFE process is proposed. Finally, the influence of the precursor structure onto the resulting nanodiamonds is discussed in detail.

\section{Experimental section}

\section{Chemicals}

1,3,5-Trinitro-1,3,5-triazine (RDX; $\mathrm{M}=222.12 \mathrm{~g} / \mathrm{mol}$ ) and 2-Methyl-1,3,5-trinitrobenzene (TNT; $\mathrm{M}=227.13 \mathrm{~g} / \mathrm{mol}$ ) were purchased by EURENCO (Massy, France). Both explosives were dried in a vacuum drying oven for $6 \mathrm{~h}$ at $50{ }^{\circ} \mathrm{C}$ before further use. Acetone (for HPLC, $\geq 99.9 \%$ ) was sourced by Sigma-Aldrich (Munich, Germany) and used without further purification.

\section{Production of hexolite mixtures}

Diverse hexolite mixtures were produced via Spray Flash Evaporation (SFE) which is described in detail elsewhere. ${ }^{25,29}$ A flow chart of the SFE setup as well as a photography of the used SFE reactor are depicted in Figure 1. For hexolite mixture production 2 wt.\% solutions of various RDX and TNT mass fractions in $500 \mathrm{~mL}$ acetone were prepared. Thus, the mass of RDX plus 
TNT amounted $8.2 \mathrm{~g}$ in each solution. The amounts of RDX and TNT for each hexolite mixture solution are summed up in Table 1. Each solution was given into the solution storage tank of the vertical SFE - reactor. A pressure of 40 bar was applied onto the solution storage tank. Afterwards the RDX-TNT-acetone solutions were sprayed through a preheated ruby hollow cone nozzle $\left(160^{\circ} \mathrm{C}\right)$ with a diameter of $200 \mu \mathrm{m}$ into the permanently evacuated atomization chamber. During the spraying the pressure inside the reaction chamber amounted $2-5$ mbar. Final hexolite products were collected as white-yellowish powders whereby the yellowish shade increases slightly with the amount of TNT. In Table 1 the hexolite mixtures are named by the mass ratios of RDX and TNT and the first number gives the percentage of RDX and the second one the percentage of TNT (e.g. 80/20).

Table 1. Mass and amount of substance of RDX and TNT for each hexolite mixture

\begin{tabular}{|c|r|r|r|r|}
\hline \multirow{2}{*}{$\begin{array}{c}\text { hexolite mass } \\
\text { fraction } \\
\text { (RDX/TNT) }\end{array}$} & \multicolumn{2}{|c|}{ RDX } & \multicolumn{2}{c|}{ TNT } \\
\cline { 2 - 5 } & mass / g & $\begin{array}{c}\text { amount of } \\
\text { substance / } \\
\text { mmol }\end{array}$ & mass / g & $\begin{array}{c}\text { amount of } \\
\text { substance / } \\
\text { mmol }\end{array}$ \\
\hline $\mathbf{8 0 / 2 0}$ & 6.5 & 29.3 & 1.7 & 7.3 \\
\hline $\mathbf{6 0 / 4 0}$ & 4.9 & 22.1 & 3.3 & 14.3 \\
\hline $\mathbf{4 0 / 6 0}$ & 3.3 & 14.7 & 4.9 & 21.6 \\
\hline $\mathbf{2 0 / 8 0}$ & 1.7 & 7.5 & 6.5 & 28.6 \\
\hline
\end{tabular}

Production of physically mixed hexolite reference samples

A reference sample using a 60/40 hexolite mixture was prepared through physical mixing. $60 \mathrm{mg}$ of RDX nanoparticles and $40 \mathrm{mg}$ of TNT nanoparticles were transferred into a porcelain mortar and mixed carefully and pressureless with a porcelain pestle. Pure RDX and TNT nanoparticles were also prepared via SFE. In a first step $8.2 \mathrm{~g}$ (36.7 mmol) RDX, respectively $8.2 \mathrm{~g}$ (35.9 mmol) TNT were solved in acetone in order to obtain $4 \mathrm{wt} \%$ solutions. Recrystallization of pure RDX and pure TNT were performed using the same SFE set-up and settings as described above. 


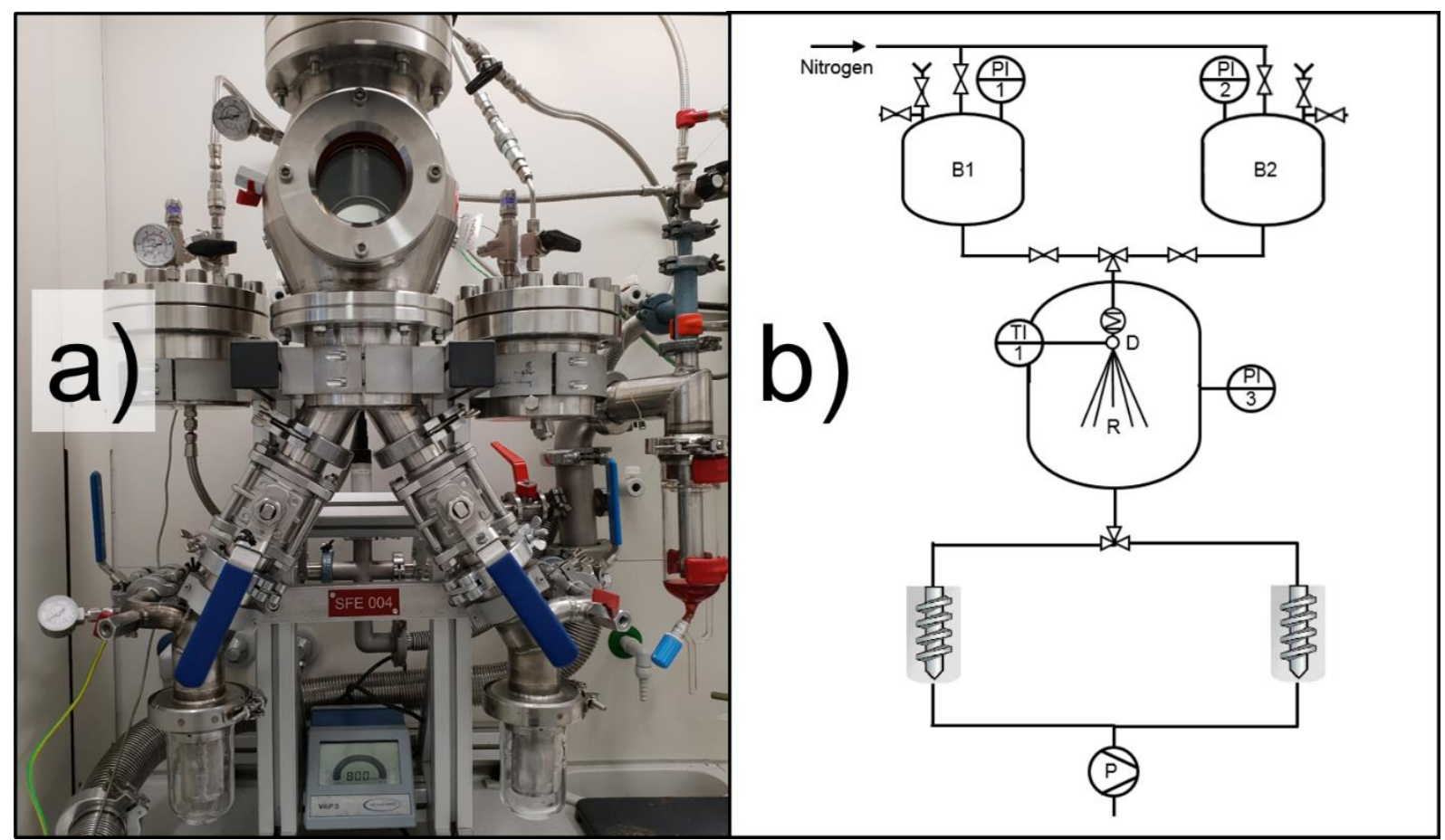

Figure 1. a) Photography of a medium sized vertical SFE - reactor. b) Flow-chart of SFE setup. Final product is collected with axial cyclones (marked in grey). B1, B2: solvent and solution tank; R: reaction chamber; D: hollow cone nozzle, P: vacuum pump; PI: pressure sensor; TIC: temperature sensor.

\section{TERS tip preparation}

AppNano ACCESS-NC AFM-probes (Mountain View, USA) were used for TERS experiments. AFM probes were mounted onto a $30^{\circ}$ prism template to generate a perpendicular arrangement between the tip and the sputtering target, since the tips are mounted onto the cantilever at an angle of $120^{\circ}$ (Figure 2.a). The prism template was inserted into the vacuum chamber of an HHV Auto 306 (Bangalore, India) argon plasma sputtering device at a distance of $10 \mathrm{~cm}$ from the sputtering target. Initially, $5 \mathrm{~nm}$ of titanium were deposited onto the tips with a deposition rate of $0.5 \AA$ s. Titanium acts as an adhesive promoter between the native oxide layer of the Si tip and Ag. Afterwards the Si-Ti tips were coated with a $25 \mathrm{~nm}$ silver layer (sputtering rate $0.5 \AA / s$ ). Finally, the TERS tips were annealed in a muffle furnace for $2.5 \mathrm{~min}$ at $320^{\circ} \mathrm{C}$ and stored under Ar and used within two days. The SEM image in Figure 2.b) shows the successful formation of a single Ag nanoparticle on the tip apex in the described procedure. $^{32}$ 

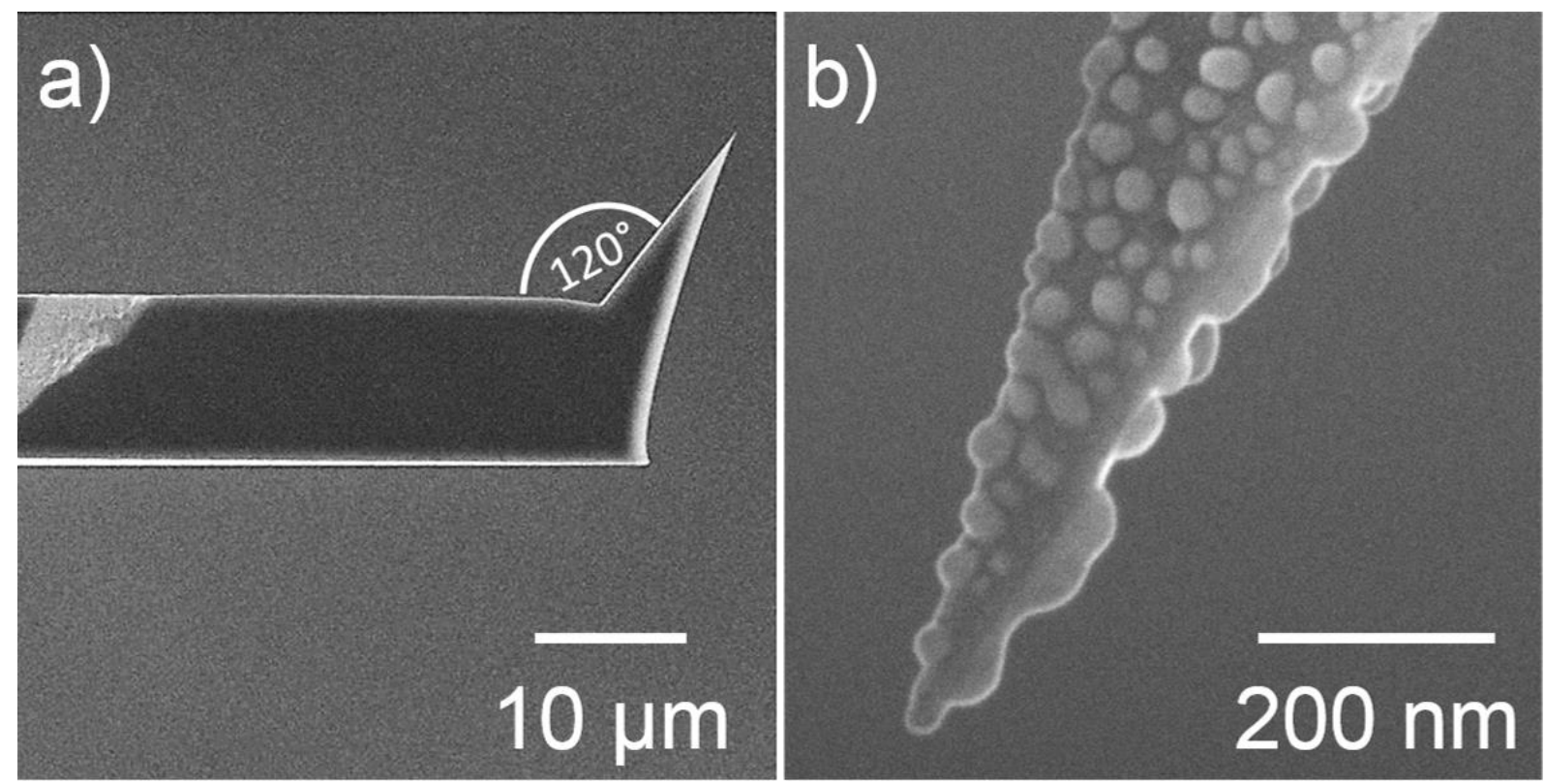

Figure 2. a) Pristine AppNano Access-NC AFM probe. The tip is mounted onto the cantilever at an angle of $120^{\circ}$.

b) $\mathrm{Si}$ - tip sputtered with a $5 \mathrm{~nm}$ layer of $\mathrm{Ti}$ and a $25 \mathrm{~nm}$ layer of $\mathrm{Ag}$. After the sputtering process the tips were annealed in a muffle furnace.

\section{Nanodiamond detonation syntheses}

Each SFE-produced hexolite mixture was pressed to cylinders with lengths and diameters of $15.8 \mathrm{~mm}$ at room temperature for 10 minutes. These cylinders were cemented afterwards with pyrotechnic glue. The cylindrical explosive charges were transferred into a water-filled pocket before firing in a detonation tank. Obtained nanodiamonds were dried via rotation evaporation.

\section{Analysis methods}

Confocal far-field Raman spectroscopy investigations were performed with a HORIBA (Kyoto, Japan) LabRam HR evolution confocal Raman microscope. In the experiment a spatula's tip of the diverse hexolite mixtures was applied to a glass slide. Single point spectra were obtained by sample excitation with a linear polarized illumination light from a $532 \mathrm{~nm}$ diode laser with an adjusted output power of $13.5 \mathrm{~mW}$ at an acquisition time of $3 \mathrm{~s}$ per spectrum. Raman mappings across the substrate were recorded with the same laser and output power. The acquisition time was set to $0.5 \mathrm{~s}$ per spectrum with a step size of $250 \mathrm{~nm}$ for these mappings. The laser light was focused onto the sample through a 100x, 0.9 NA objective. Raman scattered photons were collected with the same objective in back reflection geometry. Scattered light 
passed an edge filter, a confocal aperture with a diameter adjusted to $100 \mathrm{~nm}$ and a diffraction grating with 300 lines/mm before entering a deep cooled CCD camera $\left(-60^{\circ} \mathrm{C}\right)$. Confocal Raman maps were calculated and depicted using LabSpec Spectroscopy Suite 6.4.4. (HORIBA; Kyoto, Japan).

Atomic Force Microscopy (AFM) size distribution measurements of all SFE-produced hexolite mixtures and nanodiamonds were performed with an AIST-NT CombiScope ${ }^{\mathrm{TM}}$ 1000SPM Atomic Force Microscope (Novato, USA) in non-contact mode. The amplitude of the cantilever vibration was set to $10 \mathrm{~nm}$. All measurements were performed with AppNano ACCESS-NC AFM-probes (Mountain View, USA). The size of all AFM topographic images was $5 \mu \mathrm{m} \times 5 \mu \mathrm{m}$ with a resolution of $256 \times 256$ measurements points and a scanning rate of $0.6 \mathrm{~Hz} .200$ nanoparticles of each SFE-produced hexolite mixture and 200 nanodiamonds of each hexolite precursor were measured. Samples were prepared by depositing a spatula's tip of the diverse hexolite mixtures or nanodiamonds between two $2 \mathrm{~cm} \times 2 \mathrm{~cm}$ cover slips. The sample was spread on the glass surface through pressureless rubbing of the two glass slides against each other.

Tip Enhanced Raman Spectroscopy (TERS) surface maps were recorded with a combination of the AFM microscope and the confocal Raman microscope described above. The confocal Raman microscope acts in this combination as light source and spectrometer. All measurements were performed in non-contact AFM mode with an amplitude of $10 \mathrm{~nm}$ and samples were illuminated from the below (transmission mode). Incident linear polarized laser light (532 nm) was focused onto the tip with by a 100x, 1.4 NA oil-immersion objective. The precise positioning of the AFM-tip in the focused laser spot was achieved by objective scans perpendicular and parallel towards the AFM-TERS tip. A typical square-shaped TERS surface map consists of $100(10 \mathrm{x} 10)$ single TERS spectra. The acquisition time was $3 \mathrm{~s}$ per measurement point at a laser power on the sample of $54 \mu \mathrm{W}$. Step sizes between the measurement points depend on the size and surface area of the investigated nanoparticle and varied between 2-5 nm. The same samples were used for TERS investigations as for AFM size distribution measurements.

High Resolution Transmission Electron Microscopy (HR-TEM) images of obtained nanodiamonds were recorded on a JEOL ARM2000F (Tokyo, Japan) microscope with a 
nominal point resolution of $0.8 \AA$ at Scherzer defocus. The microscope was operated at $200 \mathrm{kV}$ acceleration voltage.

\section{Results and discussion}

Distinction of RDX and TNT via far-and near-field Raman Spectroscopy

In a first step, far-field Raman spectra of pristine RDX and TNT were recorded to obtain information about the structure composition of hexolite mixtures. Marker bands of each compound were chosen from these spectra. These marker band were detected in wavenumber regions were the other compound did not show a signal. The distinguishability of RDX and TNT molecules was already demonstrated in an earlier work and the same RDX and TNT marker bands were chosen. ${ }^{44}$ The RDX marker band at $882 \mathrm{~cm}^{-1}$ can be assigned to the ring breathing vibration. ${ }^{44-47}$ The TNT marker band between $1358-1371 \mathrm{~cm}^{-1}$ can be assigned to three $\mathrm{NO}_{2}$ symmetric stretching vibrations. ${ }^{44,48-49}$ Figure 3 shows the Raman spectra of RDX and TNT with marker bands highlighted in green respectively red. The complete Raman spectra and the assignment are given in the Supporting Information (S1). In confocal far-field Raman experiments maps were recorded of each hexolite mixture to obtain a first insight into the structure compositions. TERS was performed to investigate morphology, size and structure composition of the hexolite mixtures on the nanoscale. Due to its surface sensitivity, TERS is perfectly suited for surface characterization of composite nano materials as already demonstrated on 60/40 hexolite nanoparticles and on CL-20/HMX nano co-crystals in previous studies. $^{32,44}$ TERS surface maps each containing 100 TERS spectra $(10 \times 10$ spectra $)$ with step sizes between 2-5 $\mathrm{nm}$ (depending on the nanoparticle size) were recorded. 15 nanoparticles of each hexolite mixture were investigated. Thus, differences between far-field Raman spectra and TERS spectra of hexolite nanoparticles allowed to conclude on the distinct arrangement of RDX and TNT molecules in the particles. 


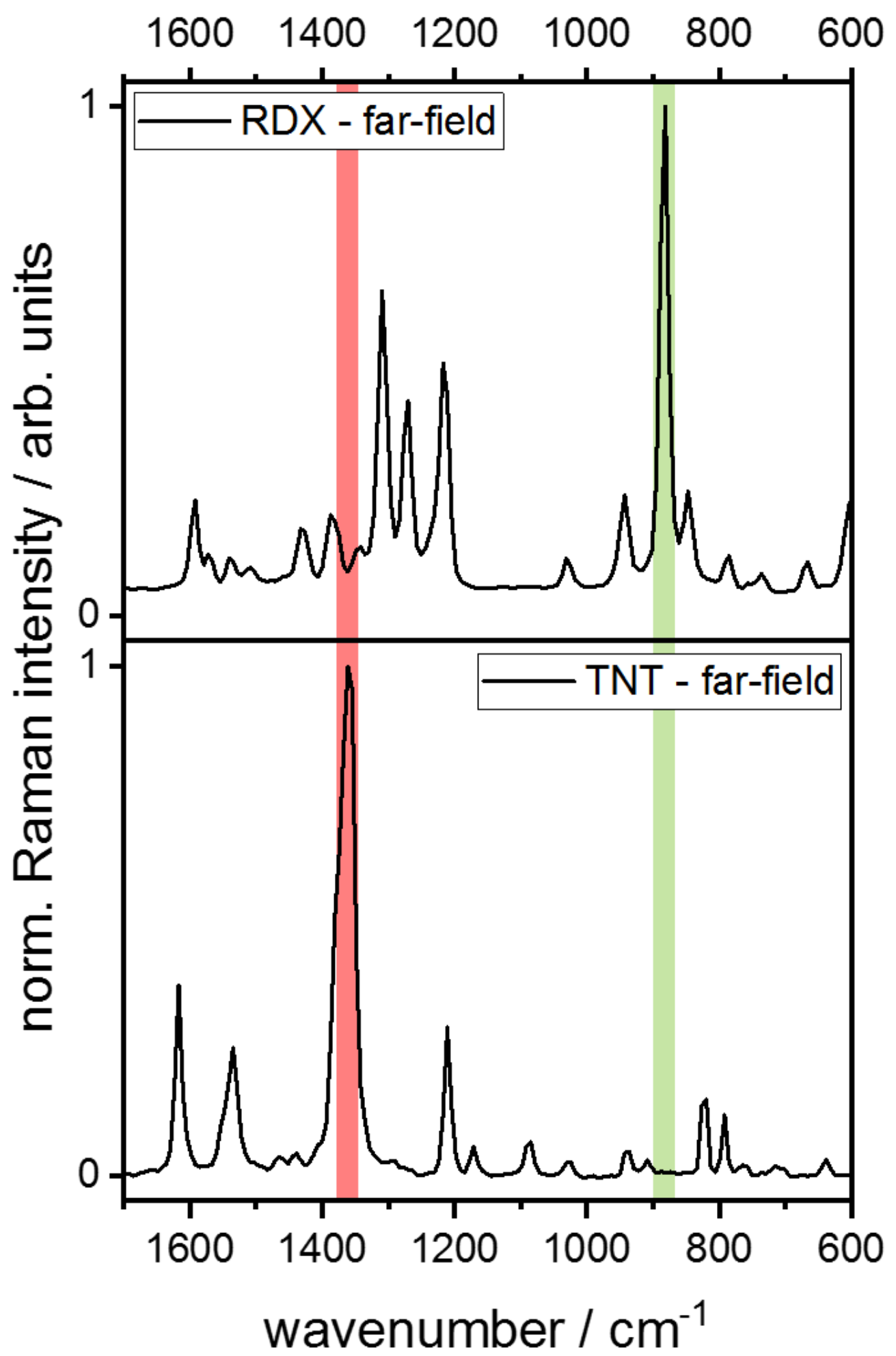

Figure 3. Far-field Raman spectra of RDX and TNT nanoparticles in the range between $1700 \mathrm{~cm}^{-1}$ and $600 \mathrm{~cm}^{-1}$. The RDX marker band $\left(882 \mathrm{~cm}^{-1}\right.$; ring breathing) is highlighted in green. The TNT marker band $\left(1358 \mathrm{~cm}^{-1}-\right.$ $1371 \mathrm{~cm}^{-1}$; three $\mathrm{NO}_{2}$ symmetric stretching vibrations) is marked in red. 


\section{Physically mixed 60/40 hexolite}

Physically mixed 60/40 hexolite (p-60/40) was received as white-yellowish powder. Confocal Raman maps of this powder were recorded to provide insight into the intermixture of RDX and TNT nanoparticles. Figure 4 shows a microscope image and the corresponding confocal Raman map of p-60/40. These confocal Raman maps were recorded pixel-wise. The Raman intensity of the RDX marker band in the single spectra is shown in green and the Raman intensity of TNT is shown in red. If both marker bands were detected simultaneously, the band intensities are superimposed and appear as light green over yellow to dark orange pixels. Evidently, the confocal Raman maps demonstrate that RDX and TNT nanoparticles consist of microscale agglomerates without a further intermixture on the nanoscale. In order to visualize this finding Figure 4.a) und Figure 4.b) present only the intensity of RDX respectively TNT marker band. Even though confocal far-field Raman maps already exclude a nanoscale intermixture of RDX and TNT nanoparticles, TERS measurements were performed on single nanoparticles to verify this result and to receive reference data for TERS investigation of SFE produced nano hexolite mixtures. As expected, only pure RDX and pure TNT nanoparticles could be identified in the
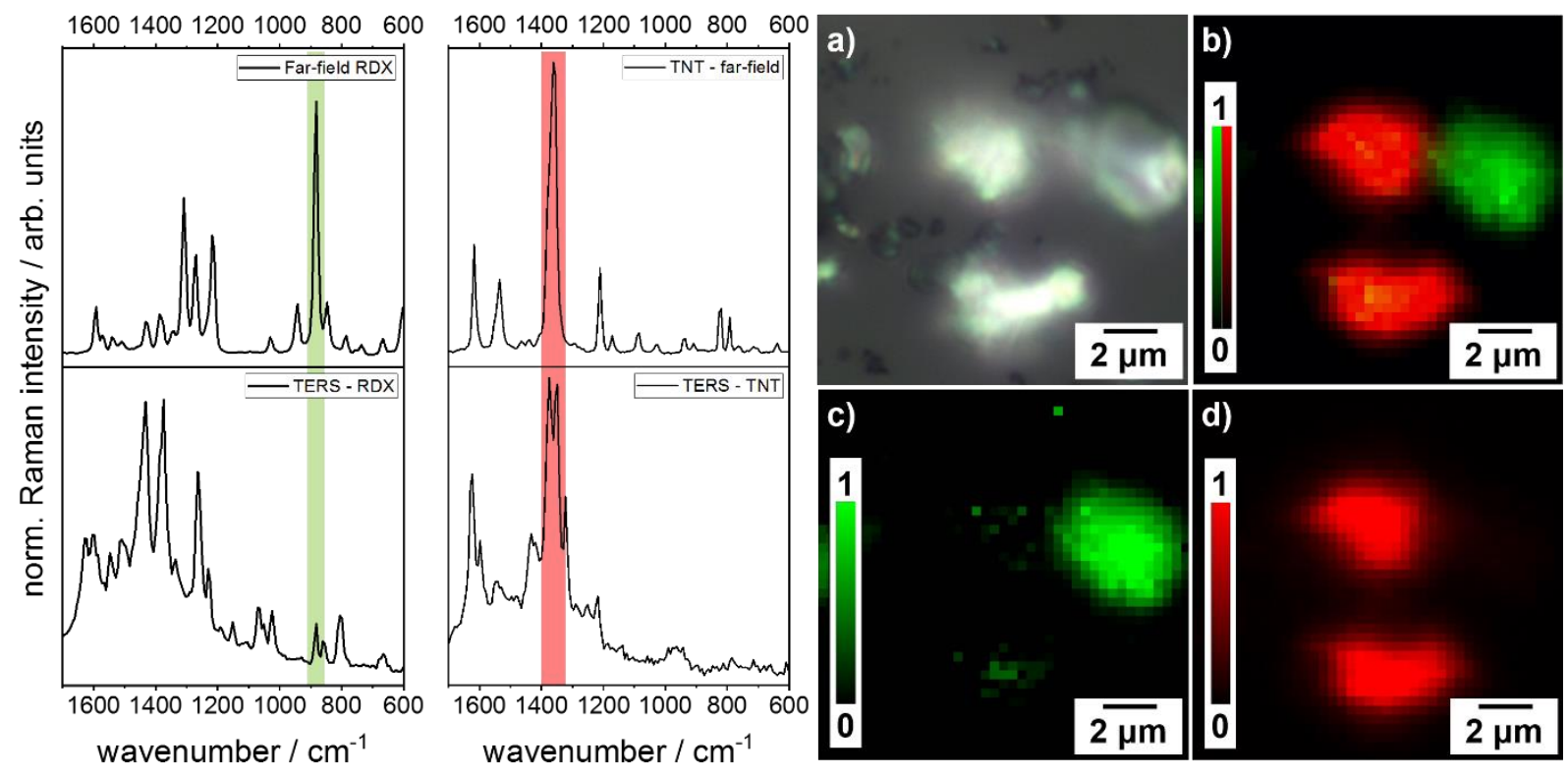

Figure 4. Left: Far-field Raman and TERS spectra of pristine RDX and TNT. Right: Confocal Raman microscopy maps of physically mixed 60/40 hexolite particles. A better comparability of the Raman intensities was achieved by normalization. a) Microscope image of p-60/40 nano hexolite particles. b) Corresponding confocal Raman map of superimposed RDX $\left(882 \mathrm{~cm}^{-1}\right.$; ring breathing, $\mathrm{NNO}_{2}$ stretching) and TNT $\left(1358 \mathrm{~cm}^{-1}-1371 \mathrm{~cm}^{-1} ;\right.$ three $\mathrm{NO}_{2}$ symmetric stretching vibrations) marker band Raman intensities. c) Confocal Raman map of RDX marker band Raman intensities. d) Confocal Raman map of TNT marker band Raman intensities. 
TERS spectra. Generally, TERS spectra differ from far-field Raman spectra by several features. Thus slight shifts of band positions and differences in Raman intensities of same vibrational modes are evident in TERS spectra (Figure 4). are evident in TERS spectra (Figure 4). Since TERS is a surface sensitive detection technique, the orientation of molecules on the probed particles plays an important role. The surface molecules are supposed to have different molecular environments due to the crystal symmetry break at the particle's surface. This environmental change is associated with a change of intermolecular interactions. ${ }^{32}$ The different molecular arrangements result in slight changes of the molecular energy levels and thus, molecular vibrations can be detected with slightly shifted band positions in TERS spectra. ${ }^{50}$ This phenomenon is comparable with Raman frequency shifts in Raman spectra of different organic crystal polymorphs. ${ }^{51-53}$ Furthermore, interactions between surface molecules and the silver coated tip may contribute to a band position shift as described above. ${ }^{38,54}$ In contrast, the far-field Raman spectra of the bulk material contain averaged information from all molecule orientations. Discrepancies of Raman intensities of same vibrational modes between far- and near-field Raman spectra can be explained by the orientation between illuminated molecules towards the electric field vector of the enhanced electric field. In general normal modes appear more intense if the vibrational direction and the electric field vector are aligned parallel. ${ }^{35,40,55-}$ ${ }^{56}$ However, TERS spectra of p-60/40 nanoparticles contain all relevant peaks of RDX or TNT and can be consequently easily assigned to the specific compound (Figure 4). The RDX marker band is detected between $870-890 \mathrm{~cm}^{-1}$ in TERS spectra of RDX nanoparticles. Also, the TNT marker band is well identifiable in a wavenumber range between $1350 \mathrm{~cm}^{-1}$ and $1375 \mathrm{~cm}^{-1}$. The shape of this broad peak can differ in TERS spectra compared to far-field Raman spectra since it contains several symmetric $\mathrm{NO}_{2}$ stretching vibrations which can be enhanced differently due to specific orientations as described above. In contrast to nano hexolite mixtures produced by SFE only six particles p-60/40 were investigated by TERS, since a molecular intermixture of RDX and TNT could be already excluded by confocal Raman microscopy. Thus, a statistical more reliable investigation was not necessary at this point. However, three RDX and three TNT nanoparticles could be clearly identified by TERS.

\section{0/20 SFE hexolite mixture}

The ultrafine yellowish 80/20 hexolite powder produced by SFE (SFE-80/20) contains nanoparticles with a mean size of $65.7 \mathrm{~nm} \pm 27.6 \mathrm{~nm}$. The particle mean sizes of all investigated hexolite mixtures were determined via AFM microscopy and are presented in the Supporting 
Information (S2). In contrast to physically mixed nano hexolite p-60/40 particles, not a single Raman spectrum containing only RDX or TNT signals could be found within SFE-80/20 confocal Raman maps. These measurements reveal that the particles are composed of an RDX/TNT mixture arrangement. The Raman mapping results are given in Figure 5. Especially confocal Raman maps showing only RDX and TNT marker band, respectively, (Figure 5.c) and d) indicate the presence of either of the compounds. On the other hand, TERS spectra of the SFE-80/20 nanoparticle surface maps indicate either an RDX/TNT mixture or pure TNT. In other words, 13 of 15 TERS surface maps of investigated SFE-80/20 nanoparticle surfaces contain RDX and TNT marker bands whereby 2 of 15 TERS spectra show only the TNT marker band. If the band positions of the $\mathrm{NO}_{2}$ modes in RDX and TNT in the TERS spectra containing both marker vibrations are compared with those of pristine RDX and TNT in the far-field Raman spectra band position shifts are recognizable (see Table 2). Since some $\mathrm{NO}_{2}$ modes of
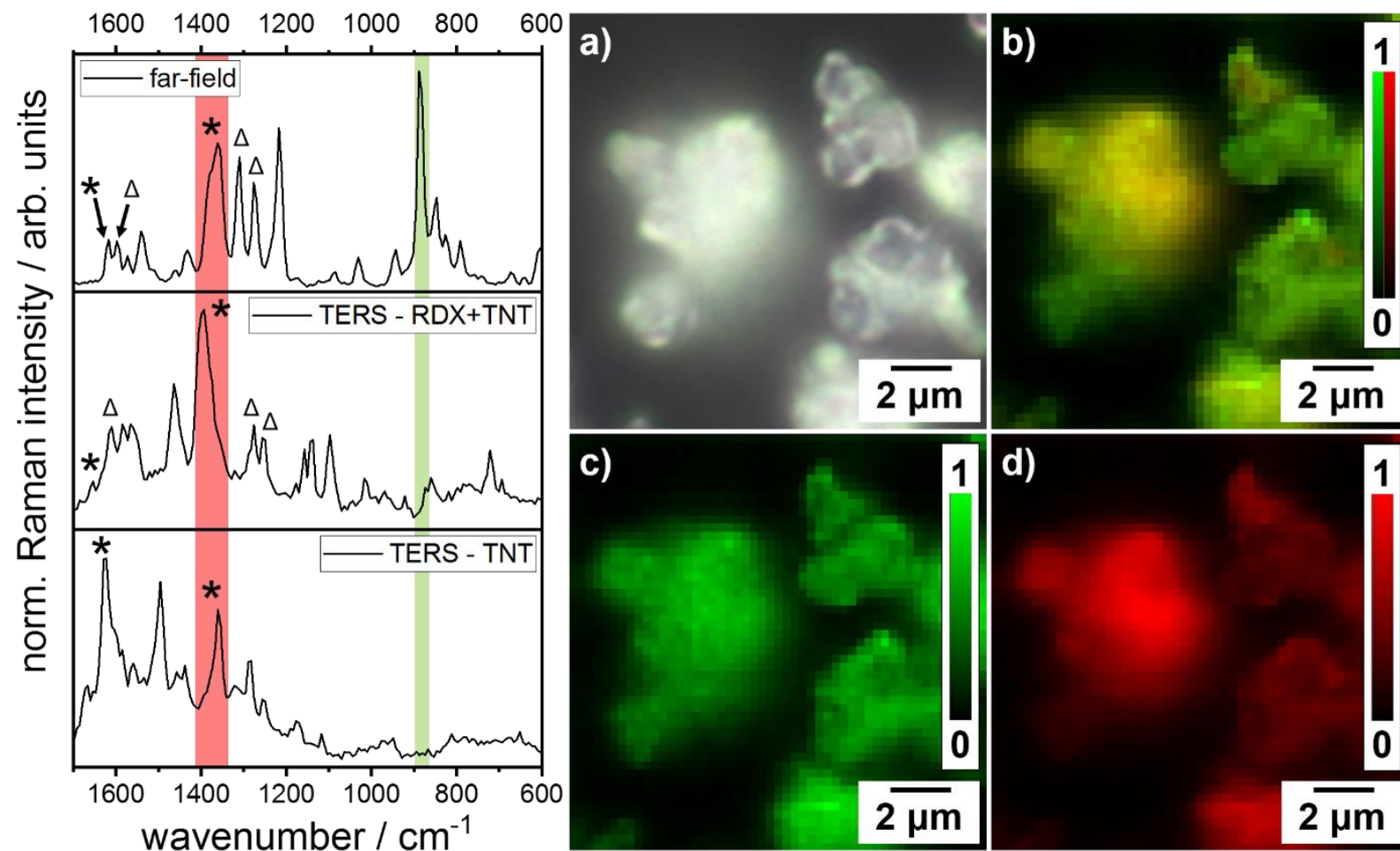

$2 \mu \mathrm{m}$

Figure 5. Left: Far-field Raman and TERS spectra of 80/20 hexolite powder produced by SFE. Typical TERS spectra show either both RDX (882 $\mathrm{cm}^{-1}$; ring breathing) and TNT $\left(1358 \mathrm{~cm}^{-1}-1371 \mathrm{~cm}^{-1}\right.$; three $\mathrm{NO}_{2}$ symmetric stretching vibrations) marker bands or only the TNT marker band. $\Delta$ indicate the $\mathrm{N}^{-\mathrm{NO}_{2}}$ stretching vibration and the $\mathrm{NO}_{2}$ asymmetric stretching vibration of RDX; * indicate the investigated $\mathrm{NO}_{2}$ stretching vibrations of TNT.

Right: Confocal Raman microscopy maps of SFE-80/20. Raman intensities are normalized for a better comparability. a) Microscope image of physically SFE-80/20 nano hexolite. b) Corresponding confocal Raman map of superimposed RDX and TNT marker band Raman intensities. c) Confocal Raman map of RDX marker band Raman intensities. d) Confocal Raman map of TNT marker band Raman intensities. 
RDX and TNT overlap in the TERS spectra, only clearly assignable vibrational modes are used for detailed analysis. In RDX two $\mathrm{N}-\mathrm{NO}_{2}$ stretching vibrations are localized at $1270 \mathrm{~cm}^{-1}$ and $1309 \mathrm{~cm}^{-1}$, respectively, and one $\mathrm{NO}_{2}$ is localized at $1592 \mathrm{~cm}^{-1}$ in SFE-80/20 far field spectra. The RDX N-NO 2 stretching vibrational modes appear slightly shifted within a wavenumber range between $1256 \mathrm{~cm}^{-1}$ and $1283 \mathrm{~cm}^{-1}$ respectively $1296 \mathrm{~cm}^{-1}$ and $1322 \mathrm{~cm}^{-1}$ in SFE-80/20 TERS spectra. The RDX $\mathrm{NO}_{2}$ asymmetric vibration can be found between $1585 \mathrm{~cm}^{-1}$ and $1622 \mathrm{~cm}^{-1}$. The TNT marker band containing three clearly identifiable Raman active $\mathrm{NO}_{2}$ symmetric stretching vibrations (between $1358 \mathrm{~cm}^{-1}$ and $1371 \mathrm{~cm}^{-1}$ in far-field Raman spectra) appears shifted between $1354 \mathrm{~cm}^{-1}$ and $1399 \mathrm{~cm}^{-1}$ in TERS spectra. The TNT NO 2 asymmetric stretching vibration localized at $1617 \mathrm{~cm}^{-1}$ in far-field Raman spectra is shifted between $1617 \mathrm{~cm}^{-1}$ and $1653 \mathrm{~cm}^{-1}$ in near-field TERS spectra. Generally, RDX N-NO 2 vibrations appear with a shift of $\pm 14 \mathrm{~cm}^{-1}$ less shifted than the $\mathrm{RDX} \mathrm{NO}$ asymmetric stretching vibration, which are detected within $+30 \mathrm{~cm}^{-1}$. The TNT symmetric and asymmetric stretching vibrations show a wavenumber shift of up to $31 \mathrm{~cm}^{-1}$ and $36 \mathrm{~cm}^{-1}$, respectively. Described shifts are supposed to appear due to intermolecular interactions between the $\mathrm{NO}_{2}$ functional groups of $\mathrm{RDX}$ on the one hand and $\mathrm{NO}_{2}$ functional groups of TNT on the other hand. Since the RDX N-NO vibrations seem to be less affected by these interactions than the $\mathrm{RDX} \mathrm{NO}_{2}$ asymmetric stretching vibration, it is supposed that negatively charged oxygen atoms of the RDX $\mathrm{NO}_{2}$ functional groups interact electrostatically with positively charged nitrogen atoms of the TNT $\mathrm{NO}_{2}$ functional groups. If the electrostatic interaction would appear between the positively charged nitrogen atoms of the $\mathrm{RDX} \mathrm{NO}_{2}$ functional group and the negatively charged oxygen atoms of the TNT $\mathrm{NO}_{2}$ functional groups, shifts of $\mathrm{RDX} \mathrm{N-NO}$ stretching vibrations are expected stronger than shifts of TNT and RDX $\mathrm{NO}_{2}$ stretching vibrations. The described intermolecular electrostatic interactions force RDX respectively TNT molecules in specific molecular arrangements which differ from their arrangements in the bulk material. Thus, vibrational modes are slightly shifted in the TERS spectra compared to far-field Raman spectra. Due to the fact that only RDX/TNT mixed surfaces and pure TNT surfaces could be identified within the TERS spectra, it is concluded that TNT nano-patches grow on RDX particle surfaces in the SFE process. This assumption is supported by the lower solubility of RDX in acetone than TNT. Thus, in SFE RDX nanoparticles are formed followed by the attachment of TNT molecules. $^{44}$ 
Table 2. Band positions of $\mathrm{NO}_{2}$ vibrational modes of RDX and TNT detected in the far-field Raman and TERS spectra

\begin{tabular}{|c|c|c|}
\hline vibration [ref. ${ }^{44-46,48-49}$ ] & $\begin{array}{c}\text { far-field Raman } \\
\text { wavenumber } / \mathrm{cm}^{-1}\end{array}$ & $\begin{array}{c}\text { TERS } \\
\text { wavenumber } / \mathrm{cm}^{-1}\end{array}$ \\
\hline $\mathrm{RDX}: \mathrm{N}-\mathrm{NO}_{2} \mathrm{str}$ & 1270 & $1256-1283$ \\
\hline $\mathrm{RDX}: \mathrm{N}-\mathrm{NO}_{2}$ str & 1309 & $1296-1322$ \\
\hline RDX: $\mathrm{NO}_{2}$ asym str & 1541 & $1527-1546$ \\
\hline RDX: $\mathrm{NO}_{2}$ asym str & 1573 & $1565-1584$ \\
\hline $\mathrm{RDX}: \mathrm{NO}_{2}$ asym str & 1592 & $1585-1622$ \\
\hline TNT: $3 \mathrm{x} \mathrm{NO}_{2}$ sym str & $1358-1371$ & $1354-1399$ \\
\hline TNT: $\mathrm{NO}_{2}$ asym str & 1534 & $1540-1546$ \\
\hline TNT: $\mathrm{NO}_{2}$ asym str & 1553 & $1559-1572$ \\
\hline TNT: $\mathrm{NO}_{2}$ asym str & 1617 & $1617-1653$ \\
\hline
\end{tabular}

asym: asymmetric; sym: symmetric; str: stretching; investigated RDX vibrations are marked in green; investigated TNT vibrations are marked in red

\section{0/40 SFE hexolite mixture}

60/40 hexolite (SFE-60/40) nanoparticles produced in SFE were collected as a yellowish powder. SFE-60/40 are composed of spherical nanoparticles with a mean size of $32.2 \mathrm{~nm} \pm$ $12.3 \mathrm{~nm}$. Confocal Raman microscopy of SFE-60/40 points to a similar RDX/TNT mixture found for SFE-80/20. The TERS experiments show that none of the 15 investigated SFE-60/40 nanoparticles surfaces is built up by RDX only. In contrast to SFE-80/20, the TERS surface investigations of SFE-60/40 particles reveal that 10 of 15 nanoparticle surfaces consist of TNT only whereas 5 of 15 TERS maps of SFE-60/40 surfaces contain both RDX and TNT marker bands. Similar results were earlier reported by Deckert-Gaudig et al. using a different TERS setup. ${ }^{44}$ The authors demonstrated that the surfaces of 5 out of 8 SFE-60/40 nanoparticles were dominated by a TNT contribution. RDX and TNT marker bands were found in 3 of 8 SFE-60/40 TERS maps. By comparing far-field Raman and TERS spectra it was concluded that RDX cores were encapsulated by TNT shells. In the case of SFE-60/40 TERS maps showing RDX and TNT marker bands it is supposed that the thickness of the TNT shells is only a few nanometers. 
Thus, vibrations of TNT surface molecules and RDX molecules close to particle surfaces are identified in the TERS spectra. TERS spectra containing both RDX and TNT marker bands show similar band position shifts as already discussed in the SFE-80/20 section above and point to similar RDX-TNT intermolecular interactions. A typical TERS spectrum containing only TNT signals and a typical TERS spectrum with RDX and TNT marker bands in SFE-60/40 nanoparticles are shown in Figure 6.
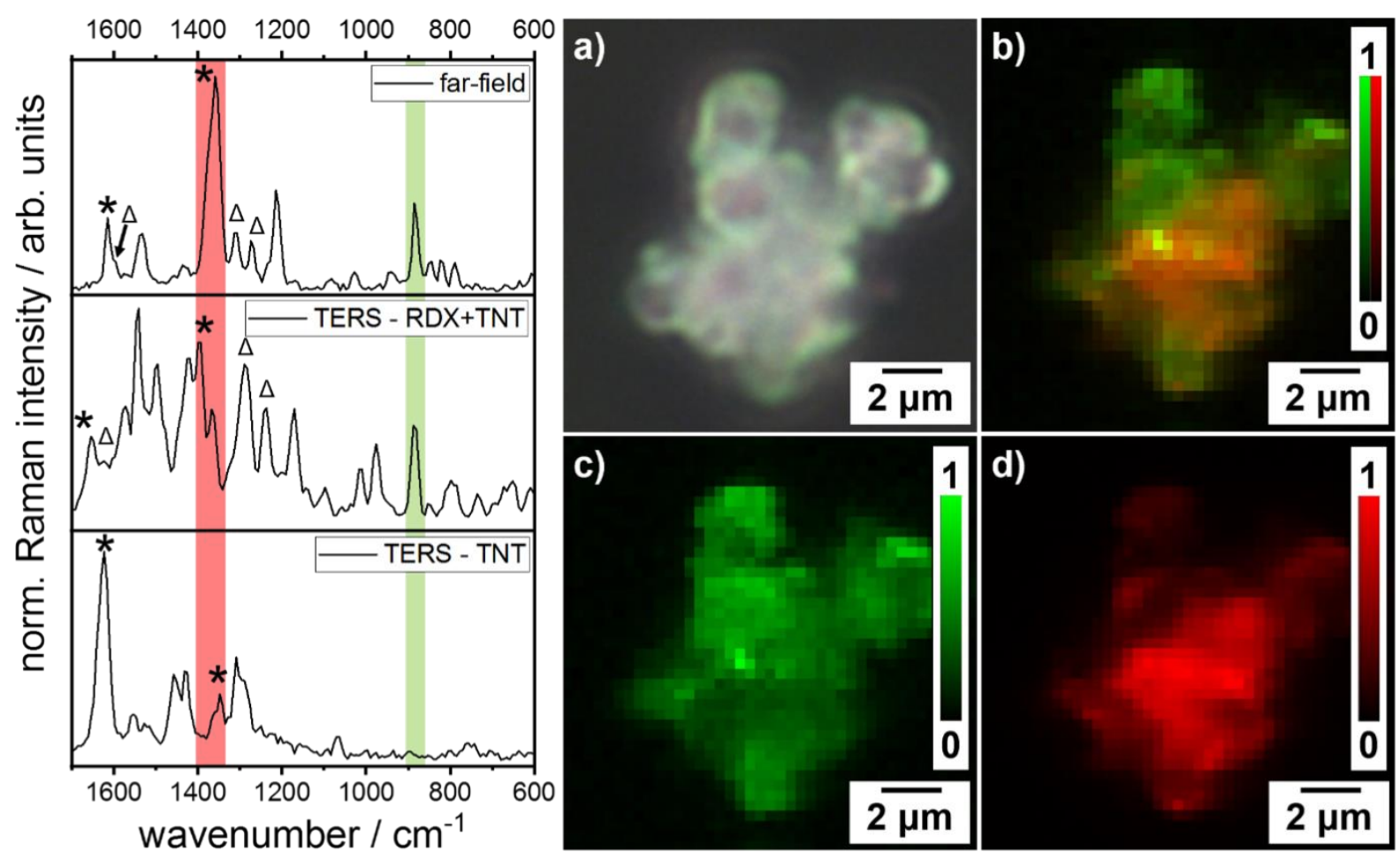

d)

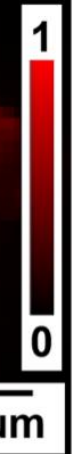

Figure 6. Left: Far-field Raman and TERS spectra of 60/40 hexolite powder produced by SFE. Typical TERS spectra show either both RDX (882 cm $\mathrm{cm}^{-1}$; ring breathing) and TNT $\left(1358 \mathrm{~cm}^{-1}-1371 \mathrm{~cm}^{-1}\right.$; three $\mathrm{NO}_{2}$ symmetric stretching vibrations) marker bands or only the TNT marker band. $\Delta$ indicate the $\mathrm{N}-\mathrm{NO}_{2}$ stretching vibration and the $\mathrm{NO}_{2}$ asymmetric stretching vibration of RDX; * indicate the investigated $\mathrm{NO}_{2}$ stretching vibrations of TNT. Right: Confocal Raman microscopy maps of SFE-60/40. Raman intensities are normalized for a better comparability. a) Microscope image of physically SFE-60/40 nano hexolite. b) Corresponding confocal Raman map of superimposed RDX and TNT marker band Raman intensities. c) Confocal Raman map of RDX marker band Raman intensities. d) Confocal Raman map of TNT marker band Raman intensities. 
40/60 SFE hexolite mixture

RDX/TNT (SFE-40/60) hexolite particles with a 40/60 weight ratio was produced via SFE as ultrafine yellowish powder. The nanoparticles have a mean size of $29.4 \mathrm{~nm} \pm 11.8 \mathrm{~nm}$. All far-field Raman spectra of SFE-40/60 show RDX and TNT marker bands (see Figure 7). The TERS analysis reveals that 12 of 15 investigated SFE-40/60 particle surfaces contain a TNT shell. In contrast, 3 out of 15 TERS maps show RDX and TNT marker bands. At first glance, these results appear unexpected since an increased amount of TNT is supposed to form thicker TNT shells around the RDX cores. As a result, RDX molecules should be undetectable by the electromagnetic field at the TERS tip apex. However, 3 out of 15 TERS maps demonstrate the presence of RDX molecules localized close to or even on SFE-40/60 nanoparticle surfaces. At this point, the formation of isotropic formed hierarchically ordered RDX/TNT core/shell nanoparticles appeared rather implausible. However, the mixed RDX/TNT TERS spectra show
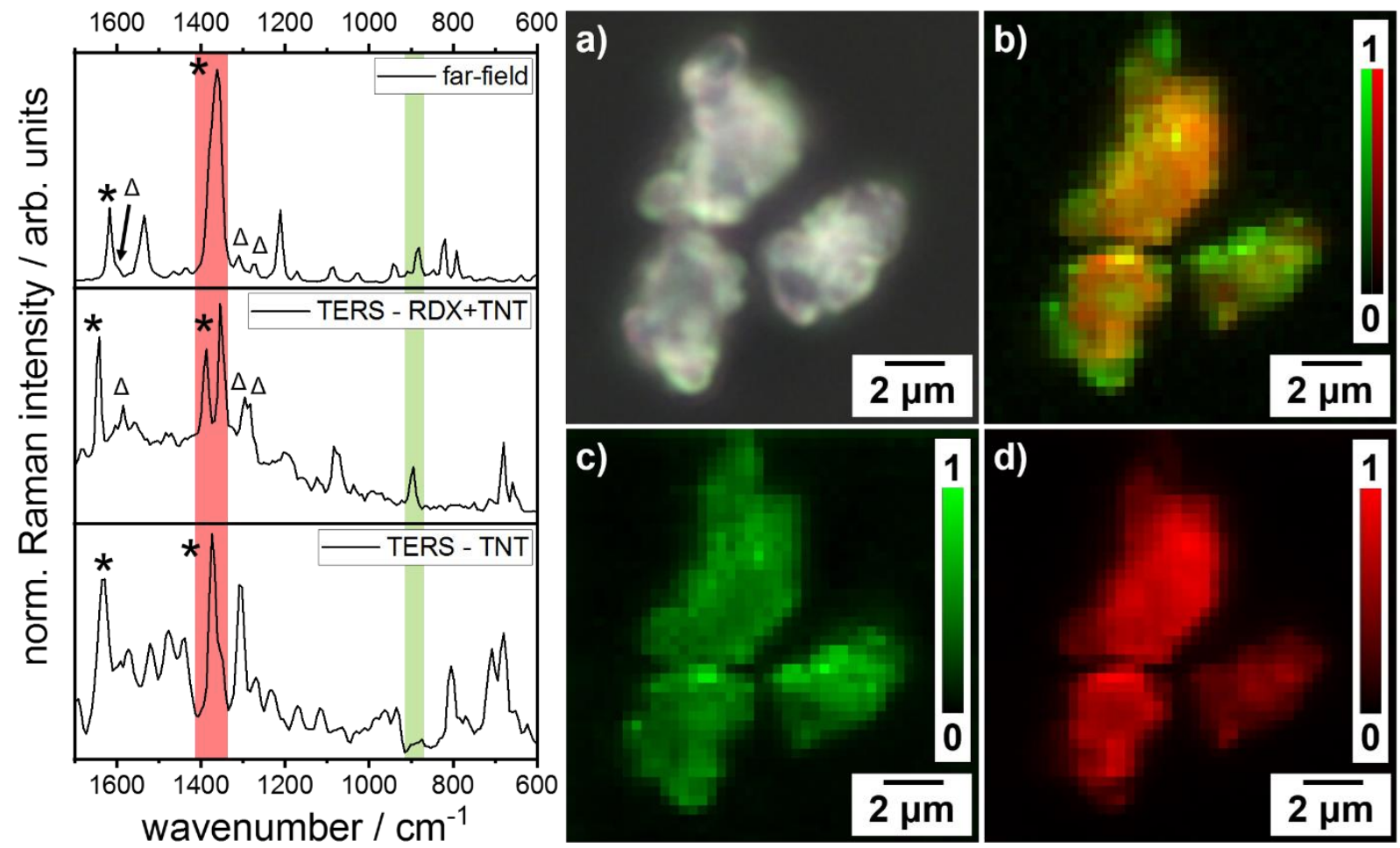

Figure 7. Left: Far-field Raman and TERS spectra of 40/60 hexolite powder produced by SFE. Typical TERS spectra show either both RDX (882 cm-1; ring breathing) and TNT $\left(1358 \mathrm{~cm}^{-1}-1371 \mathrm{~cm}^{-1}\right.$; three $\mathrm{NO}_{2}$ symmetric stretching vibrations) marker bands or only the TNT marker band. $\Delta$ indicate the $\mathrm{N}^{-\mathrm{NO}_{2}}$ stretching vibration and the $\mathrm{NO}_{2}$ asymmetric stretching vibration of RDX; * indicate the investigated $\mathrm{NO}_{2}$ stretching vibrations of TNT. Right: Confocal Raman microscopy maps of SFE-40/60. Raman intensities are normalized for a better comparability. a) Microscope image of physically SFE-40/60 nano hexolite. b) Corresponding confocal Raman map of superimposed RDX and TNT marker band Raman intensities. c) Confocal Raman map of RDX marker band Raman intensities. d) Confocal Raman map of TNT marker band Raman intensities. 
the same band position shifts of RDX and $\mathrm{TNT} \mathrm{NO}_{2}$ modes indicating similar intermolecular interactions between RDX and TNT molecules at the particle surfaces. A typical TERS spectrum showing only TNT signals and a typical TERS spectrum containing RDX and TNT marker bands of SFE-40/60 nanoparticles are given in Figure 7.

\section{0/80 SFE hexolite mixture}

A 20/80 hexolite mixture (SFE-20/80) was collected as an ultrafine yellowish powder composed of nanoparticles with a mean size of $59.7 \mathrm{~nm} \pm 23.8 \mathrm{~nm}$. In agreement with the farfield Raman measurements of the other SFE hexolite mixtures, all far-field Raman spectra contain RDX and TNT marker bands. The confocal Raman map given in Figure 8 illustrates these results by the pixel-wise superimpositions of RDX and TNT marker bands intensities. TERS maps reveal that 13 out of 15 SFE-20/80 nanoparticle surfaces are formed of TNT only.
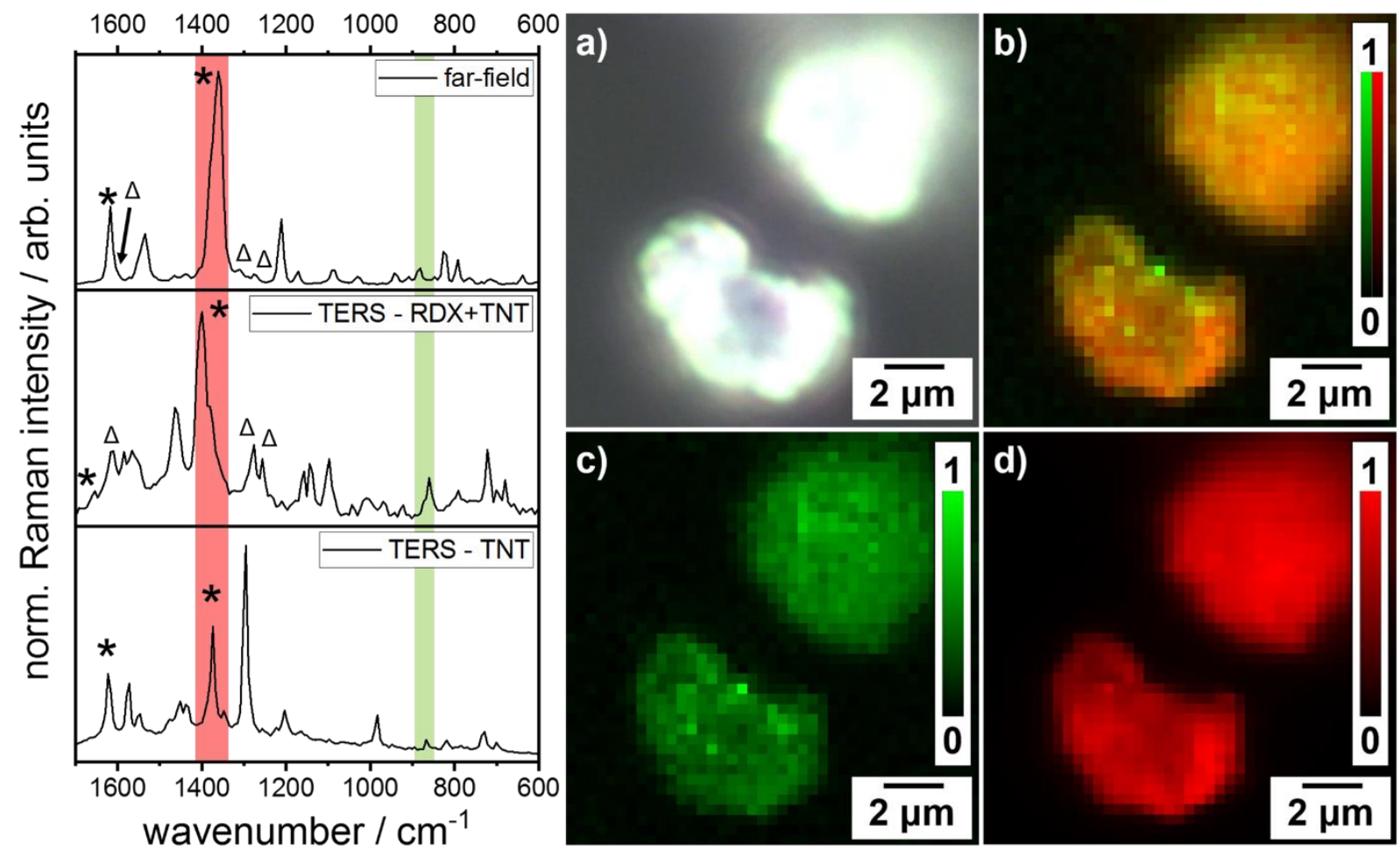

Figure 8. Left: Far-field Raman and TERS spectra of 20/80 hexolite powder produced by SFE. Typical TERS spectra show either both RDX (882 $\mathrm{cm}^{-1}$; ring breathing) and TNT $\left(1358 \mathrm{~cm}^{-1}-1371 \mathrm{~cm}^{-1}\right.$; three $\mathrm{NO}_{2}$ symmetric stretching vibrations) marker bands or only the TNT marker band. $\Delta$ indicate the $\mathrm{N}^{-\mathrm{NO}_{2}}$ stretching vibration and the $\mathrm{NO}_{2}$ asymmetric stretching vibration of $\mathrm{RDX}$; * indicate the investigated $\mathrm{NO}_{2}$ stretching vibrations of TNT. Right: Confocal Raman microscopy maps of SFE-20/80. Raman intensities are normalized for a better comparability. a) Microscope image of physically SFE-20/80 nano hexolite. b) Corresponding confocal Raman map of superimposed RDX and TNT marker band Raman intensities. c) Confocal Raman map of RDX marker band Raman intensities. d) Confocal Raman map of TNT marker band Raman intensities. 
TERS spectra from 2 out of 15 SFE-20/80 hexolite nanoparticles contain RDX and TNT marker bands. Although SFE-20/80 contains four times more TNT than RDX, RDX molecules are still detectable in the TERS spectra. This observation strengthens the assumption that SFE produced hexolite mixtures are not formed by isotropic RDX/TNT core/shell nanoparticles. Similar band position shifts of RDX and TNT $\mathrm{NO}_{2}$ functional group vibrations could be identified within SFE-20/80 TERS spectra as like in TERS spectra of the other hexolite composite nanoparticles. An example TERS spectrum containing both RDX and TNT marker bands and an example TERS spectrum including only TNT marker bands are presented in Figure 8. . Since the number of surfaces containing TNT only increases proportionally with the TNT mass fraction while the RDX marker band can still be detected in some TERS spectra of each measured hexolite mixture, an anisotropic RDX/TNT core/shell formation is suggested. The proposed building mechanism of such anisotropic core/shell nanoparticles will be discussed in detail in the next section.

\section{Building mechanism of hierarchically structured hexolite nanoparticles}

The combination of AFM, confocal Raman microscopy and TERS results provide insights into the size, morphology and molecular composition of different hexolite mixtures. The smallest nanoparticle mean size of $29.4 \mathrm{~nm} \pm 11.8 \mathrm{~nm}$ was found for SFE-40/60 hexolite nanocomposites. Particle mean sizes increase with increasing the mass fraction of RDX or TNT within the hexolite precursor solutions. Thus, SFE-80/20 nanoparticles having a diameter of $65.7 \mathrm{~nm} \pm 27.6 \mathrm{~nm}$ provide the largest particle mean size. SFE-60/40 shows a particle mean size of $32.3 \mathrm{~nm} \pm 12.3 \mathrm{~nm}$ and SFE-20/80 a mean size of $59.7 \mathrm{~nm} \pm 23.8 \mathrm{~nm}$. The dependence of particle mean sizes is presented as a function of the RDX mass fraction in Figure 9.a). Since all recorded far-field Raman spectra of each SFE produced hexolite mixtures contain RDX and TNT marker bands, confocal far-field Raman microscopy and spectroscopy demonstrates that RDX and TNT molecules are mixed on the nanoscale during the SFE process. Furthermore, TERS surface analysis demonstrates that not a single nanoparticle of all SFE produced hexolite nanoscale composites contains RDX only. Thus, TERS maps of all measured hexolites particles point either to surface covered by TNT only or to a mixture of RDX and TNT. The number of particles covered by TNT only increases with an increase of the TNT mass fraction. In contrast, the number of hexolite nanoparticle surfaces formed by RDX and TNT decreases consistently with an increased TNT mass fraction (Figure 9.b). Moreover, frequency shifts of $\mathrm{NO}_{2}$ 
vibrations are detectable in TERS spectra containing both RDX and TNT marker bands. These shifts point towards an interaction between the $\mathrm{NO}_{2}$ substituents in RDX and TNT.

In order to describe the building mechanism of the diverse hexolite mixtures it is necessary to briefly consider the dynamics of the SFE process. During the SFE process a pressured acetone solution is sprayed through a hollow cone nozzle into a permanently evacuated reaction chamber. By passing the hollow cone nozzle the precursor solution is accelerated and transformed into a fine spray characterized by droplets with a mean size of $2.6 \mu \mathrm{m} \pm 0.8 \mu \mathrm{m}$ (measured $6 \mathrm{~mm}$ behind the nozzle, measurement details are presented in the Supporting Information S3). Leaving the hollow cone nozzle, parts of acetone droplet surfaces are in contact with the ruby surface of the nozzle. It is supposed that at these interfaces the free electron pairs of the carbonyl group in acetone form hydrogen bonds with hydrogen atoms of hydroxide ions determining the ruby surface. Since only localized areas of the acetone droplets surfaces interact with the nozzle surface, droplets are accelerated inhomogeneously while leaving the nozzle. Because of the inhomogeneous acceleration and turbulences within the SFE crystallization chamber acetone droplets start to spin (Figure 9). Additionally, to the translational and angular acceleration forced by the nozzle, acetone droplets are continuously accelerated while passing the reaction chamber due to the flow provided by the vacuum pump. During the whole SFE processes the droplet size shrinks consistently until the solvent is completely evaporated (see Supporting Information, S3). Thus, the droplets act as accelerated dynamic micro reactors in the SFE process. For a better comprehensibility the building mechanisms for patchy SFE-80/20 nanoparticles and SFE-60/40, SFE-40/60, SFE-20/80 core/shell nanoparticles are described firstly without considering the acceleration of the dynamic micro-reactor droplets.

Directly after the injection of the hexolite solution droplets into the evacuated reaction chamber, the concentrations of RDX and TNT molecules within these microscale droplets are still the same as it is in the previously prepared acetone solutions. The continuous evaporation of acetone during SFE process and the related droplet shrinking results in a consistent increase of the RDX and TNT concentrations within the microscale droplets until the solution is supersaturated with RDX molecules. The supersaturation level of RDX molecules within the hexolite solution droplets is reached first since RDX exhibits with $6.8 \mathrm{~g}$ in $100 \mathrm{~g}$ acetone a sixtime less solubility than TNT with $42.4 \mathrm{~g}$ per $100 \mathrm{~g}$ acetone at room temperature. ${ }^{44}$ Following the classical theory of nucleation and crystallization, RDX molecules organizes to nuclei while 
a)

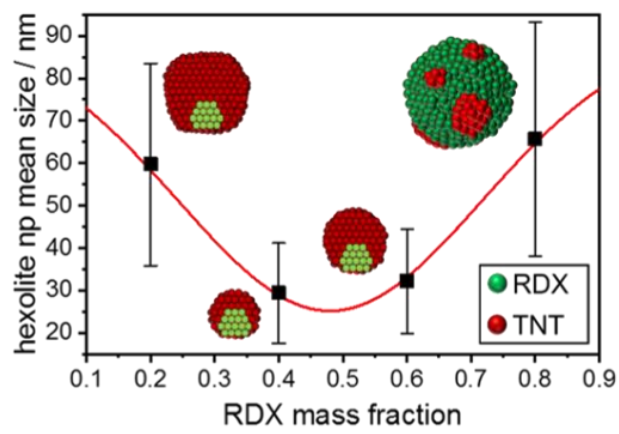

b)

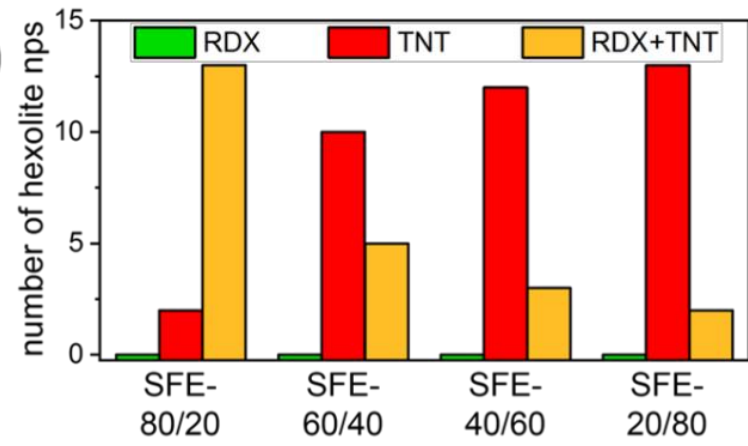

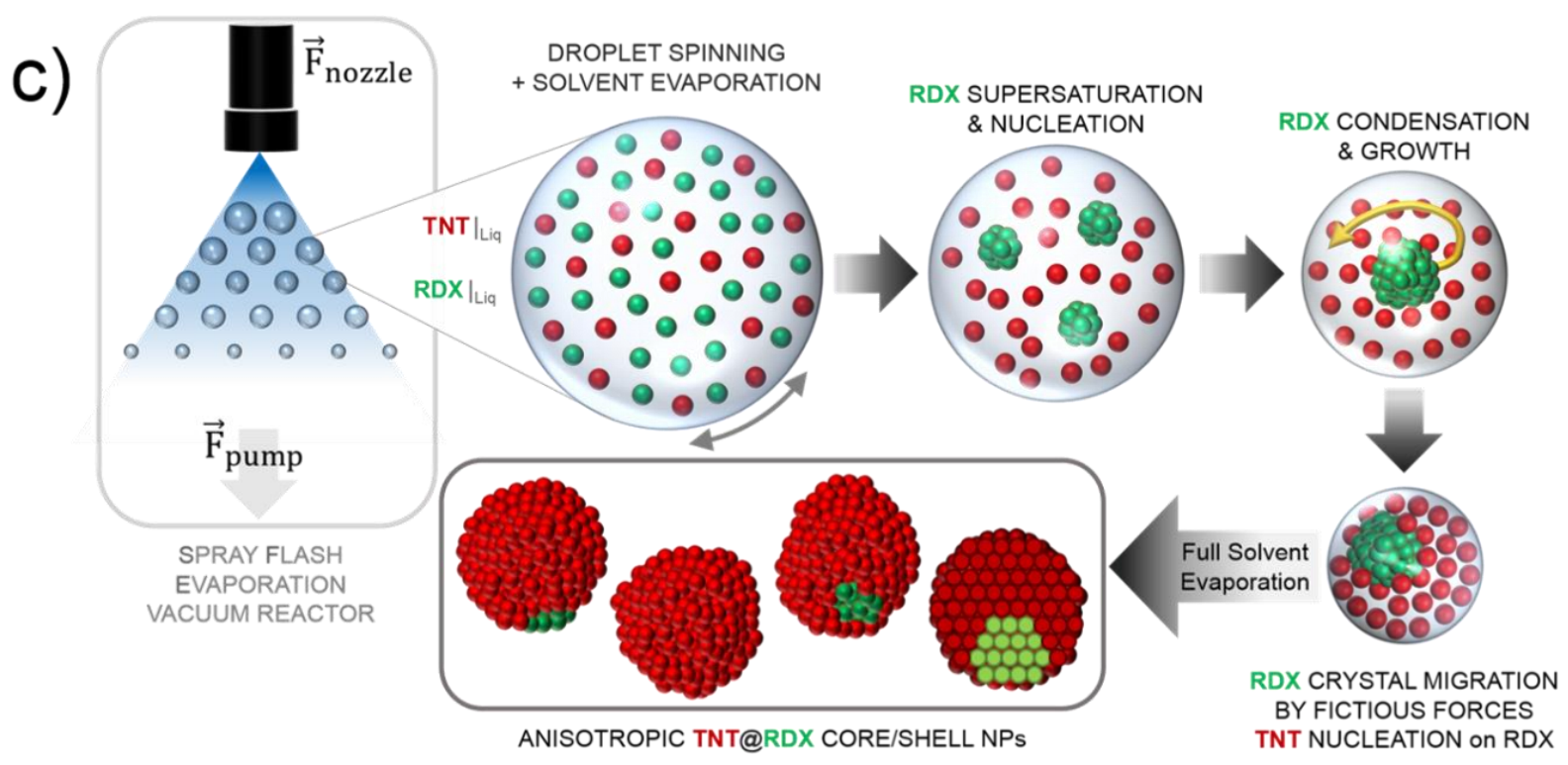

Figure 9. a) Mean sizes of anisotropic hexolite core/shell nanoparticles (SFE-80/20, SFE-60/40, SFE-40/60) and patchy hexolite sub-micron particles (SFE-20/80) as a function of the RDX mass fraction inside the precursor solutions. Green spheres represent RDX molecules; red spheres symbolize TNT molecules. Anisotropic hexolite core/shell nanoparticles are depicted as cross sections aimed to visualize its core/shell structure. b) Numbers of anisotropic hexolite nanoparticles and patchy hexolite sub-micron particles providing RDX only, TNT only and RDX/TNT mixed surfaces. c) Flowchart of the supposed anisotropic RDX/TNT core/shell nanoparticle building mechanism.

the concentrations of RDX and TNT molecules further increase (Figure 9.c). ${ }^{57}$ These RDX nuclei grow to RDX nanoparticles. The growth of RDX nuclei to RDX nanoparticles appear due to the attaching of solved RDX molecules onto RDX nuclei, the aggregation of various RDX nuclei and nanoparticles and further due to Ostwald ripening. The driving force behind the nuclei growth and aggregation to nanoparticles is the minimization of surface energy which is realized by decreasing the surface-area-to-volume ratio. Anyway, nucleation and crystallization of RDX occurs first in all produced hexolite nano-composites independent of the used RDX and TNT mass fractions within the precursor solution. Due to further shrinking of 
the droplets and the related increase of RDX and TNT concentrations within the droplets the point of supersaturation of TNT in the solution is reached in the next step. Consequently, TNT molecules start to nucleate on top of RDX nanoparticle surfaces acting as nucleation seeds. As discussed within the SFE-80/20 section, the physisorption of at least the first layer of TNT molecules onto RDX nanoparticles surfaces is supposed to be initiated by electrostatic interactions between positively charged nitrogen atoms and negatively charged oxygen atoms of the $\mathrm{NO}_{2}$ functional groups of both RDX and TNT molecules. Further TNT molecules grow onto this first TNT spots during the continued SFE process leading to the formation of the investigated TNT shells or TNT patches until the complete acetone is evaporated and the hexolite nanoparticles can be collected within the filter system (Figure 9.c). However, due to the different RDX and TNT mass fractions used within the precursor solutions, hexolite nano-composites differ in size and arrangement. While mainly TNT only determined surfaces could be identified in TERS surface maps of SFE-60/40, SFE-40/60 and SFE-20/80 hexolite nanoparticles, TERS maps of SFE-80/20 surfaces contain dominantly both RDX and TNT marker bands. As already mentioned before, these results indicate the formation of RDX/TNT core/patches or Janus like nanoparticles for SFE-80/20. In contrast, SFE-60/40, SFE-40/60 and SFE-20/80 nano-composites are formed by hierarchal ordered nanoparticles providing an RDX/TNT core/shell character. Since the nucleation and crystallization of RDX occurs first, a certain amount of TNT molecules is needed to cover the primary RDX nanoparticles completely with a TNT shell. In case of SFE-80/20, the point of TNT supersaturation within the dynamic micro reactor droplets is reached later compared to the other hexolite systems due the relatively low TNT mass fraction (20\%). Thus, RDX molecules are allowed to organize themselves to larger primary particles within the droplets before TNT molecules start to adsorb onto RDX nanoparticle surfaces. Since symmetric and asymmetric stretching vibrations of $\mathrm{NO}_{2}$ functional groups of TNT appear less shifted within TERS spectra of TNT only determined hexolite surfaces, it is supposed that intermolecular interactions between TNT molecules within the TNT shell and the TNT patches are rather comparable with the intermolecular interactions in TNT crystals. Thus TNT molecules are supposed to grow preferred onto previously adsorbed TNT molecules instead of uncoated RDX surfaces since intermolecular TNT-TNT interactions (electrostatic interactions between $\mathrm{NO}_{2}$ functional groups and $\pi$ - $\pi$ stacking) should appear stronger than TNT-RDX interactions (electrostatic interactions between $\mathrm{NO}_{2}$ functional groups only) leading to a reduction of surface and Gibbs free energy. The mechanism of these island or patch formations might be comparable with the Vollmer-Weber growth describing the formation of adatom clusters due to stronger adatom-adatom interactions than adatom-surface 
interactions. ${ }^{58}$ Anyway, larger mean sizes of RDX primary particles result in the described SFE-80/20 patchy or Janus like particle structure since the amount of TNT molecules is insufficient to guarantee a complete surface coating. Contrary, SFE-60/40, SFE-40/60 and SFE-20/80 hexolite mixture provide a sufficient TNT content to form RDX/TNT core/shell particles. In accordance to the building mechanism of SFE-80/20 patchy nanoparticles, the formation of primary RDX nanoparticles takes place in a first step within these systems. These primary RDX nanoparticles appear smaller than for SFE-80/20 due to the lower mass fraction of RDX within the hexolite precursor solutions allowing a complete TNT surface coverage. While the TNT concentration increases within the droplets due to the related droplet size shrinking, RDX cores are coated with a TNT shell following the previously described mechanism. Additionally, further RDX core growths are quenched by the TNT encapsulations. Up to now, the presented reaction mechanism allows to explain the occurrence of different particle structure and sizes. However, the building mechanism does not include an explanation why TERS surface maps of RDX/TNT core/shell nanoparticles still include RDX signals even if the mass fraction of TNT amounts $80 \%$ yet. Estimations of the RDX core diameters and the TNT shell thicknesses reveal that a typical SFE-60/40 core/shell nanoparticle provides an RDX core with a diameter of $26.7 \mathrm{~nm}$ and a TNT shell thickness of $2.7 \mathrm{~nm}$. SFE-40/60 is formed by an RDX core with a diameter of $29.4 \mathrm{~nm}$ and a $4.1 \mathrm{~nm}$ thick TNT shell. The diameter of the RDX core of a typical SFE-20/80 nanoparticle amounts $59.7 \mathrm{~nm}$ and the TNT shell thickness amounts $12.9 \mathrm{~nm}$. The estimations are based on the particle mean sizes of the diverse hexolite nano-composites and the densities of crystalline RDX and TNT. The results are summarized in Table 3. The detailed calculations are given within the Supporting Information (S4).

Table 3. Estimated RDX core diameters and TNT shell thicknesses for isotropic hexolite core/shell nanoparticles

\begin{tabular}{|r|r|r|r|}
\hline $\begin{array}{c}\text { mass fraction ratio } \\
\text { (RDX/TNT) }\end{array}$ & particle mean size & RDX core diameter & TNT shell thickness \\
\hline $60 / 40$ & $32.2 \mathrm{~nm}$ & $26.7 \mathrm{~nm}$ & $2.7 \mathrm{~nm}$ \\
\hline $40 / 60$ & $29.4 \mathrm{~nm}$ & $21.2 \mathrm{~nm}$ & $4.1 \mathrm{~nm}$ \\
\hline $20 / 80$ & $59.7 \mathrm{~nm}$ & $34.0 \mathrm{~nm}$ & $12.9 \mathrm{~nm}$ \\
\hline
\end{tabular}

Due to the rapid field decay of the enhanced electric field generated by the LSPR of the silver nanoparticle localized at the AFM tip apex, the appearance of RDX signals within TERS spectra 
is at least very unlikely for SFE-60/40 and SFE-40/60 de facto impossible for SFE-20/80 (Figure 10.a). ${ }^{34}$ Therefore the contribution of RDX Raman active frequencies to diverse TERS spectra must be related to the position of the RDX cores within the hexolite RDX/TNT core/shell nanoparticles. As mentioned before, the previous building mechanism describes the hexolite nano-composite formation without considering the translational and angular accelerations of the droplets in the SFE atomization chamber. Without these accelerations it can be assumed that RDX primary nanoparticles would be located randomly within the bulk of the droplets without stronger movements until the complete evaporation of acetone is finished resulting in the formation of isotropic RDX/TNT core/shell nano-spheres with a centered RDX core. However, during SFE process droplets are accelerated due to the release of the pressurized precursor solution into the evacuated atomization chamber through a hollow cone nozzle, the interactions of acetone droplet surface molecules with the nozzle surface while leaving the nozzle, turbulences in the atomization chamber and the flow generated by the vacuum pump. The first steps of the hexolite core/shell nanoparticle building mechanism by means of RDX superstation and nucleation within the micron sized droplets take place as already described for the non-accelerated case. After the phase change of solved RDX into solid RDX nuclei and the aggregation of these RDX nuclei into primary RDX nanoparticles, the acceleration of the droplets starts to influence the formation of the hierarchical order hexolite nanoparticles. Due to the translational accelerations (by nozzle and pump, Figure 9.c) of the shrinking dynamic micro reactor droplets, a fictitious force affects the RDX primary nanoparticles pushing them back towards the edge of the droplets. This fictitious force is qualitatively the same fictitious force which presses a passenger into the seat of a car while the car is accelerated. Additionally, the inhomogeneous acceleration of the droplet while leaving the nozzle and turbulences within the SFE crystallization chamber induce a spin of the acetone micro droplets. This droplet spin is described by an angular acceleration of the droplet around itself. Consequently, a centrifugal force affects the RDX primary nanoparticles within the droplet pushing them towards the edge of the acetone droplet (Figure 9.c). The combination of both effects forces RDX primary particles to looping motions starting in the droplet bulk and ending at the droplet surface. Similar looping motions and particle migrations are described by Chung for the motion of coal particles inside accelerated n-hexane respectively methanol micro droplets. ${ }^{59}$ During the RDX nanoparticle migration the droplet sizes decrease continuously leading to TNT supersaturation and TNT nucleation on top of the RDX nanoparticles as already described for the nonaccelerated case. Since the RDX core particles are localized close to or at the edges of the acetone droplets during the growth of the TNT shell, the formation of hexolite core/shell 
nano-spheres processes anisotropic (Figure 9.c). Consequently, RDX cores are not centered in the middle of hexolite core/shell nanoparticles but are localized near or at the particle edges. Due to the varying RDX mass fractions within the different hexolite precursor solutions, kinetics of RDX nucleation and RDX core growths differ. RDX nucleation and RDX nanoparticle growth occur earlier the higher the RDX mass fraction. Accordingly, RDX primary nanoparticles are less affected by the fictitious forces the less the RDX mass fraction. Thus, a complete migration of RDX nanoparticles towards the droplets edges might not takes place for smaller RDX mass fraction hexolite systems. Therefore, it is expected that the RDX cores of SFE-20/80 and SFE-40/60 appear more centered inside hexolite nanoparticles. On the other hand, SFE-60/40 nanoparticles provide RDX cores which are located closer to the particle edges than to the particle centers. However, all of these arrangements cause nanoparticles providing partially thicker and thinner TNT shell areas. Thus, particle surface areas formed by RDX only or by RDX covered with a thin TNT layer occur. These RDX or RDX/TNT determined surface areas result in the presence of RDX Raman active frequencies within some TERS spectra of SFE-60/40, SFE-40/60 and SFE-20/80 TERS surface maps. Due to the anisotropic RDX/TNT

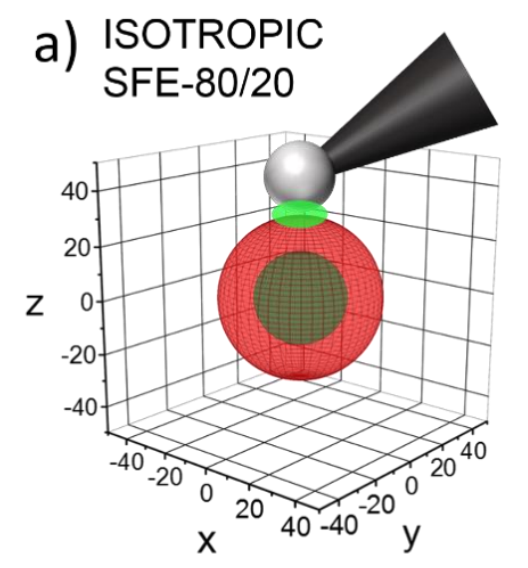

RDX core 0 TNT shell

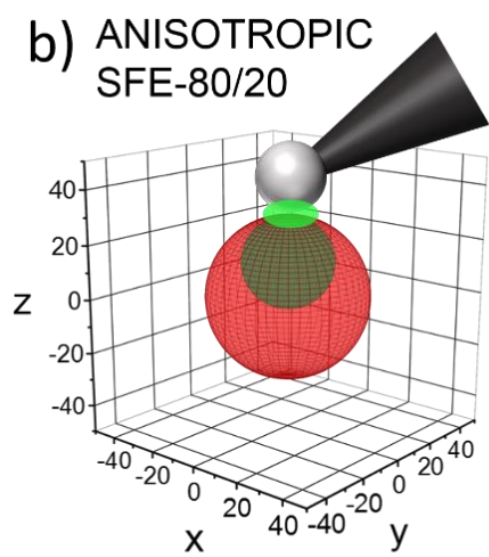

Ag NP@ @ AFM tip

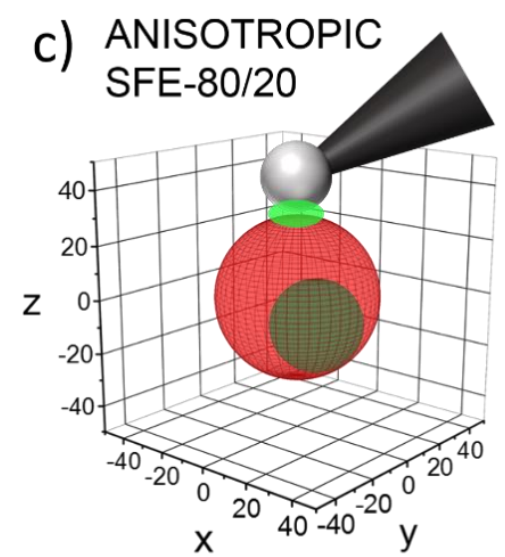

enhanced $\vec{E}$-field

Figure 10. Different alignments of SFE-80/20 RDX/TNT core/shell particles towards the silver particle mounted at the AFM tip apex. Axis units are given in nm. a) Isotropic SFE-80/20 RDX/TNT core/shell nanoparticle. Due to the closed TNT shell only TNT Raman active frequencies would occur in TERS spectra. b) Anisotropic SFE-80/20 RDX/TNT core/shell nanoparticle. The RDX core is aligned towards the silver nanoparticle localized at the AFM tip apex. RDX or RDX and TNT Raman active frequencies appear in TERS spectra. c) Anisotropic SFE-80/20 RDX/TNT core/shell nanoparticle. The RDX core is not localized in the enhanced electric field of the silver nanoparticle. Only TNT Raman active frequencies appear in TERS spectra. 
core/shell nanoparticle morphology the number of TERS spectra containing RDX signals decreases with the increase of the TNT mass fraction within the hexolite mixtures. An increase of the TNT mass fraction leads to smaller RDX cores and thicker TNT shells. Therefore, the surface area of a hexolite nanoparticle formed by RDX respectively RDX and TNT decreases. Consequently, the possibility that the particle aligns in a manner towards the silver coated AFM tip so that the RDX determined surface area contributes to the TERS spectra decreases and thus less RDX and RDX/TNT determined surfaces are found (Figure 10).

\section{Influence of the hexolite precursor size and structure onto the resulting nanodiamonds}

Reaction products of the detonation syntheses of SFE produced hexolite mixtures were collected as dark-grey ultrafine powders after drying. In order to ascertain if nanodiamonds were formed during the detonation syntheses, reaction products of each SFE produced hexolite nanoparticles were investigated via HR-TEM and TERS. HR-TEM images of each sample demonstrates clearly the formation of nanodiamonds. Figure 11 (right) depicts a sample HRTEM image of nanodiamonds synthesized from SFE-40/60 (ND-40/60). TERS spectra of nanodiamonds contain the broad graphene $\mathrm{G}$ - and D - bands localized at $\sim 1600 \mathrm{~cm}^{-1}$ respectively $\sim 1400 \mathrm{~cm}^{-1}$ and the diamond band at $1325 \mathrm{~cm}^{-1}$. The occurrence of the graphene
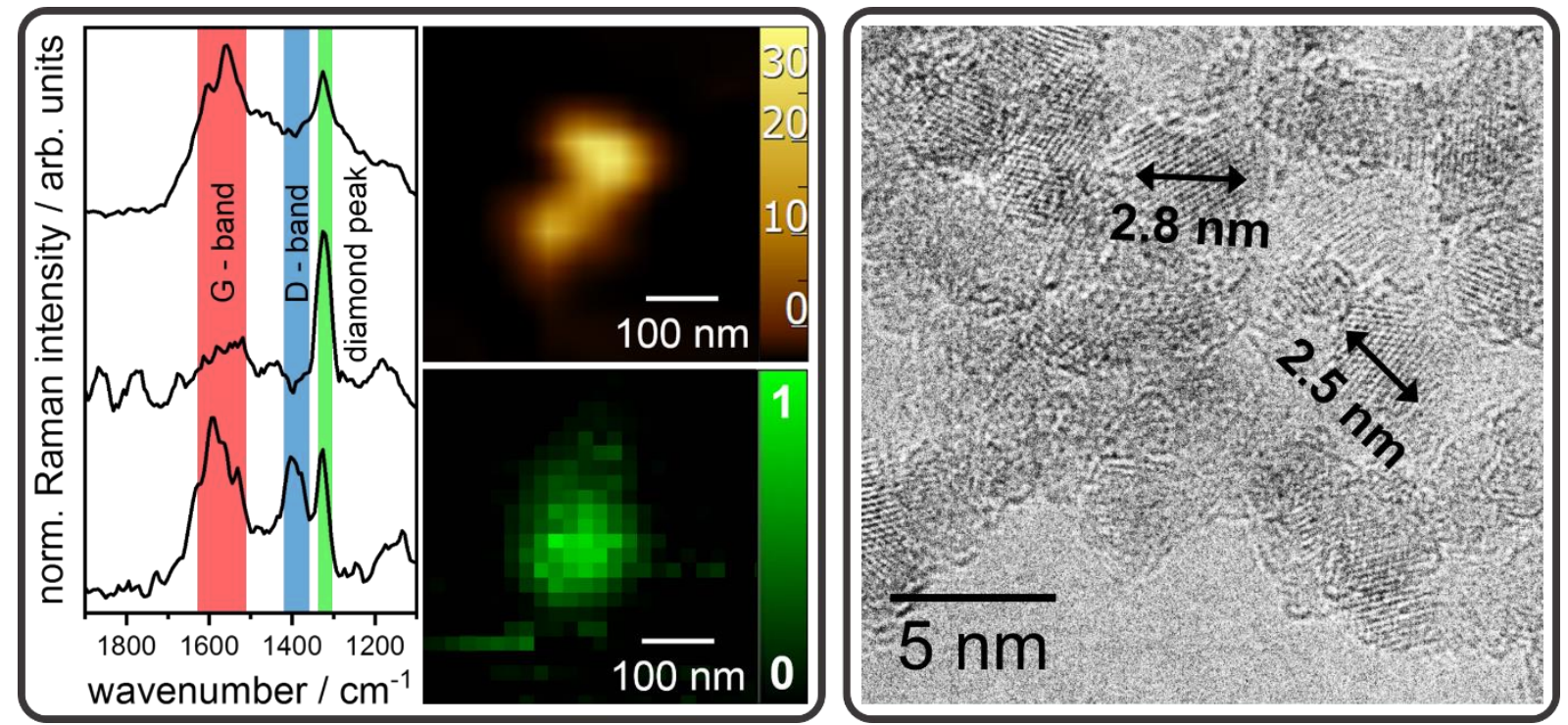

Figure 11. Left: TERS spectra of certain nanodiamonds synthesized from SFE-40/60 contain graphene G - and $\mathrm{D}$ - bands $\left(\sim 1600 \mathrm{~cm}^{-1}, 1400 \mathrm{~cm}^{-1}\right)$ as well as the diamond peak $\left(1325 \mathrm{~cm}^{-1}\right)$. The shift of the diamond peak from $1333 \mathrm{~cm}^{-1}$ to $1325 \mathrm{~cm}^{-1}$ hints onto a nanodiamonds size $<10 \mathrm{~nm}$. The AFM topographic map and the corresponding TERS map depict an agglomerate of nanodiamonds synthesized from SFE-40/60. Right: HR-TEM image of nanodiamonds synthesized from SFE-40/60. 
$\mathrm{G}$ - and D - bands within the TERS spectra points to the formation of a graphene layer on the nanodiamond surfaces. The shift of the diamond vibration from $1333 \mathrm{~cm}^{-1}$ to the measured $1325 \mathrm{~cm}^{-1}$ hints to nanodiamond sizes below $10 \mathrm{~nm}$. Presented TERS results are in good agreement with a recently published study undertaken by Ali et al. ${ }^{60}$ Since TERS measurements are performed innately on single particles the exact position of the $\mathrm{G}$ - and $\mathrm{D}$ - bands may varying between single spectra as well as the intensities of these bands and the intensity of the diamond vibration. AFM topographic maps were recorded to determine the nanodiamond means sizes and size distribution. These investigations reveal that nanodiamonds resulting from SFE-40/60 provide with $3.6 \mathrm{~nm} \pm 0.04 \mathrm{~nm}$ the smallest mean size and the narrowest size distribution. Detonation of SFE-20/80 leads to nanodiamonds (ND-20/80) with a particle mean size of $3.7 \mathrm{~nm} \pm 0.08 \mathrm{~nm}$, nanodiamonds synthesized from SFE-60/40 (ND-60/40) show a mean size of $4.1 \mathrm{~nm} \pm 0.04 \mathrm{~nm}$. SFE-80/20 result with $6.1 \mathrm{~nm} \pm 0.2 \mathrm{~nm}$ in the largest nanodiamond mean size (ND-80/20). Nanodiamond means sizes and their evolution are summed up in Figure 12 as a function of the RDX mass ratio within the precursor SFE-produced hexolite nano-composites. It has already been reported that the hexolite precursor particle sizes influences strongly the mean size and the size distribution of the resulting nanodiamonds. Pichot et al. describe a correlation between the size reduction of the precursor material from the microonto the nanoscale and a related decrease of nanodiamond mean sizes. ${ }^{23-24}$ Different hexolite and octolite (mixture of HMX and TNT) nano compounds with varying mass fraction of RDX and TNT respectively HMX and TNT are compared with micro structured hexolite and octolite mixtures in these studies. Both hexolite and octolite nano-composites were also prepared by SFE using similar settings as in the present work (see experimental section). They could demonstrate that the mean sizes of obtained nanodiamonds occur up to $50 \%$ smaller for nanostructured hexolite precursor compared to the micro structured precursors by same RDX and TNT mass fractions. Due to the lack of an accurate measurement technique which enables the investigation of the molecular arrangements of single particles on the nanoscale, they had to assume that RDX and TNT nanoparticles appear uncoupled and nearly homogeneous distributed within the pressed hexolite pellets which were prepared for nanodiamond detonation synthesis. Due to the assumed homogenous distribution of RDX and TNT particles, they proposed that the combustion reaction products of RDX (or HMX) form a diffusion barrier due to the RDX detonation pressure around single TNT nanoparticles (or more precisely the reaction products of the TNT nanoparticle) leading to a diffusion zone which is much smaller than the diffusion zone calculated for $1 \mathrm{~nm}$ nanodiamond nuclei. ${ }^{20}$ Consequently, the amount of carbon atoms arising from TNT molecules which are mainly involved in the nanodiamond 


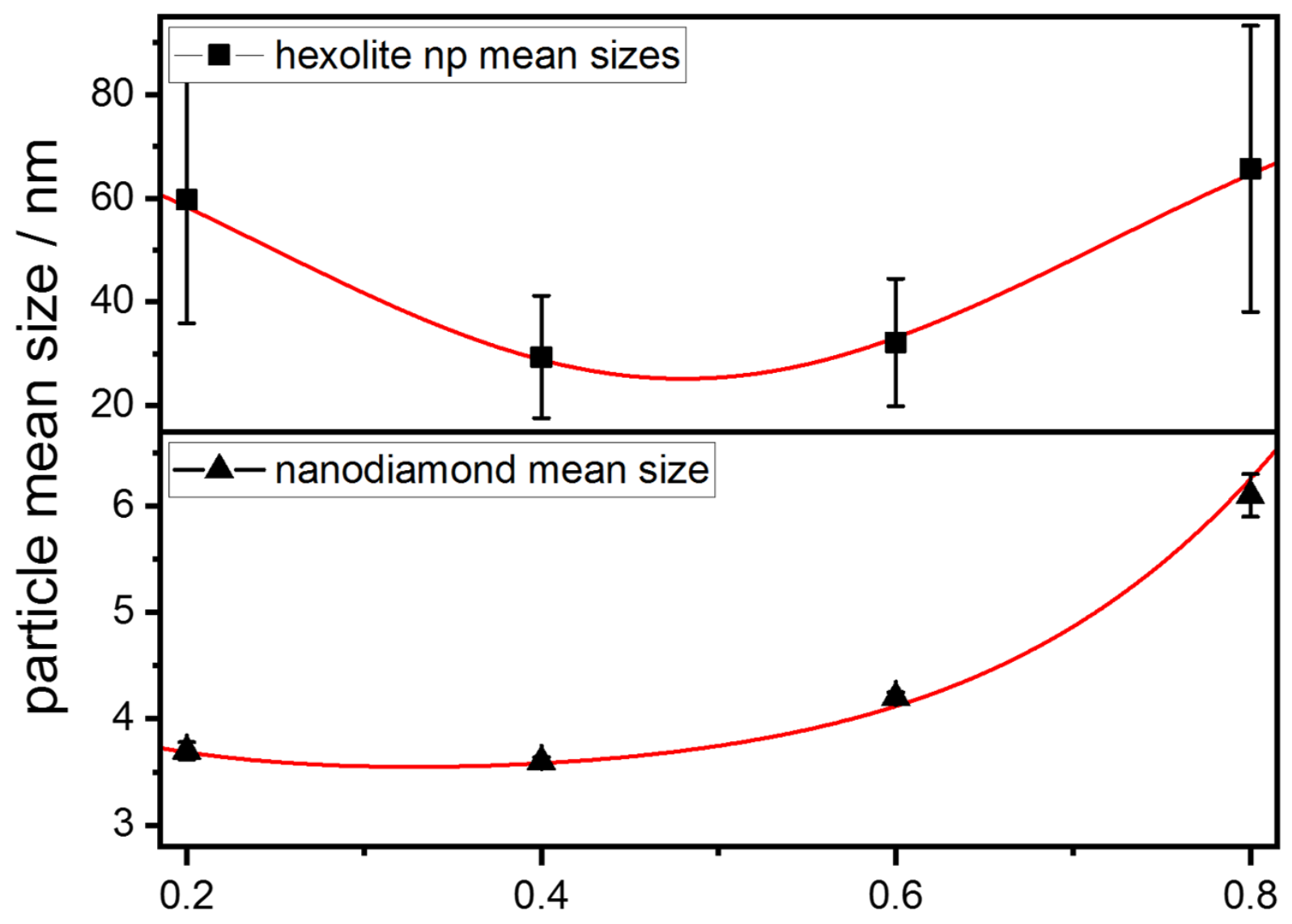

RDX mass fraction / -

\begin{tabular}{|l|r|r|r|r|}
\hline $\begin{array}{l}\text { mass fraction } \\
\text { ratio (RDX/TNT) }\end{array}$ & $80 / 20$ & $60 / 40$ & $40 / 60$ & $20 / 80$ \\
\hline$d_{\text {hex }}$ & $\begin{array}{r}65.7 \mathrm{~nm} \\
\pm 27.6 \mathrm{~nm}\end{array}$ & $\begin{array}{r}32.2 \mathrm{~nm} \\
\pm 12.3 \mathrm{~nm}\end{array}$ & $\begin{array}{r}29.4 \mathrm{~nm} \\
\pm 11.8 \mathrm{~nm}\end{array}$ & $\begin{array}{r}59.7 \mathrm{~nm} \\
\pm 23.8 \mathrm{~nm}\end{array}$ \\
\hline $\mathrm{d}_{\mathrm{nd}}$ & $\begin{array}{r}6.1 \mathrm{~nm} \\
\pm 0.20 \mathrm{~nm}\end{array}$ & $\begin{array}{r}4.2 \mathrm{~nm} \\
\pm 0.05 \mathrm{~nm}\end{array}$ & $\begin{array}{r}3.6 \mathrm{~nm} \\
\pm 0.04 \mathrm{~nm}\end{array}$ & $\begin{array}{r}3.7 \mathrm{~nm} \\
\pm 0.08 \mathrm{~nm}\end{array}$ \\
\hline
\end{tabular}

Figure 12. Evolution of hexolite RDX/TNT core/shell nanoparticles and evolution of detonation synthesized nanodiamond mean sizes as a function of the RDX mass fraction within the hexolite precursor nano-composite.

formation depends on the TNT nanoparticle diameter. Smaller TNT nanoparticles imply smaller diffusion zones which include less amounts of carbon atoms resulting generally in a decrease of nucleation sites and thus in smaller nanodiamonds. However, TERS investigations could show that SFE produced hexolite mixtures appear as anisotropic RDX/TNT core/shell particles and not as uncoupled RDX and TNT nanoparticles as presumed. Furthermore, here presented nanodiamonds do not follow this trend completely. Nanodiamonds resulting from the detonation of SFE-20/80 show a larger particle mean size than nanodiamonds produced from 
SFE-60/40 although SFE-60/40 core/shell nanoparticles provide a smaller particle mean size than SFE-20/80 nanoparticles. Consequently, the building mechanism proposed by Pichot et al. has to be slightly reconsidered.

For this purpose, an ideal case which is defined by monodispersed isotropic RDX/TNT core/shell nanoparticles forming a hexagonal close-packed precursor material after pressing is discussed first (Figure 13). After ignition and detonation, RDX reaction products are accelerated from the particle centers outwards whereas TNT reaction products are accelerated both towards the particle center and away from the particle center. The migration of RDX and TNT reaction products is driven by their specific detonation pressures. Depending on the RDX and TNT mass fractions which define the RDX core diameter and the TNT shell thickness, the competing migration effects can be dominated either by the RDX detonation pressure or by the TNT detonation pressure. Generally, RDX providing a higher detonation pressure than TNT. In case of RDX detonation pressure domination, TNT reaction products are forced into the center of a tetrahedron while RDX reaction products form a spherical diffusion barrier around it. If TNT detonation pressure dominates the competing migration effects due to a relatively low RDX mass fraction, RDX reaction products are forced to spherical diffusion barriers around the center of a tetrahedron. The tetrahedrons arise from the hexagonal close packing of the material and represents the smallest repetitive area. The sites of the tetrahedron are defined by the connection lines between the centers of two neighboring isotropic hexolite particles. Thus, four times a fourth of the TNT reaction products of one single hexolite core/shell particles contributes to one diffusion zone. Anyway, depending on whether the RDX or TNT detonation pressure dominates, different thermodynamic circumstances regarding the pressure and the temperature prevail within the diffusion zones. Since in case of the RDX detonation pressure dominance the TNT reaction products are forced towards the tetrahedron centers, pressure and temperature are higher within these resulting diffusion zones than for diffusion zones formed in a TNT dominated system. Higher pressures and temperatures promote a more complete conversion of carbon atoms into nanodiamonds leading consequently to larger nanodiamond diameters. Contrary, smaller pressure and temperature values result in a less amount of carbon atoms forming nanodiamonds and thus in smaller nanodiamond mean sizes. However, due to the high symmetry of ideal packed and perfectly monodispersed isotropic hexolite core/shell particles, the size of the diffusion zones and the thermodynamic circumstances within one diffusion zone is constant for all diffusion zones by a given RDX respectively TNT mass fraction. Consequently, the number of nucleation sites within the diffusions zones appears also constant and finally the number and sizes of obtained nanodiamonds within one diffusion zone 

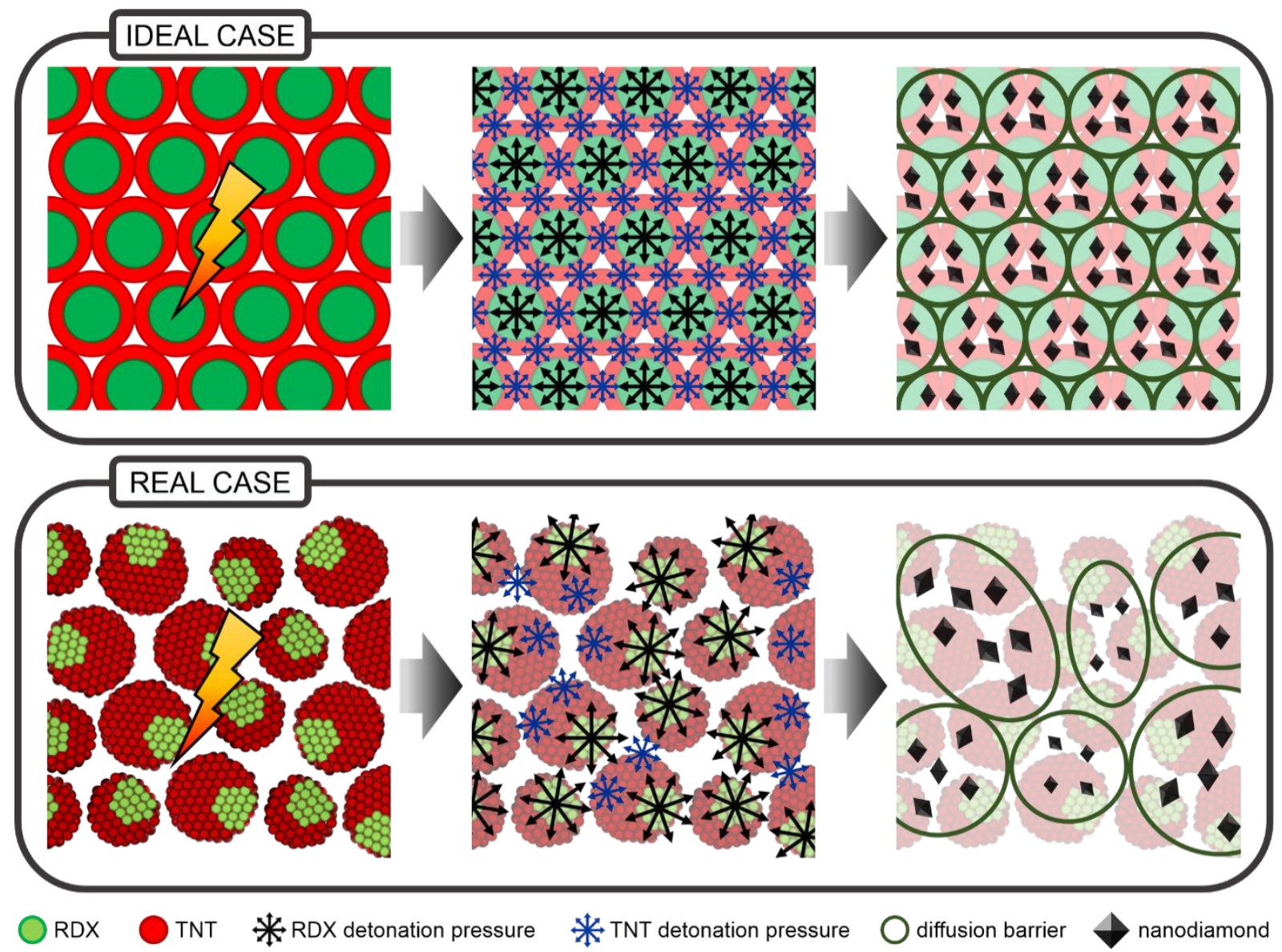

Figure 13. Top: Supposed building mechanism of nanodiamonds under ideal conditions presented as 2D cross sections. The hexolite precursor are built up by hexagonal close-packed isotropic RDX/TNT core/shell nanoparticles. Due to the higher detonation pressure of RDX compared to TNT and the high symmetry of the precursor, RDX reactions products form spherical diffusion barriers after detonation. Inside the diffusion zone nanodiamonds are formed from TNT carbon atoms. In the ideal case all diffusion zones have the identic size and contain the same amount carbon atoms and nucleation sites. Thus, the same amount of monodispersed nanodiamonds are formed within the diffusion zones. Consequently, the nanodiamond size depends only on the TNT shell thickness in the ideal case. Bottom: Supposed building mechanism of nanodiamonds under real conditions presented as 2D cross sections. SFE-produced anisotropic core/shell nanoparticles are packed dense. Due to the size distribution of the hexolite precursor particles a perfect hexagonal close packing is not possible. The random arrangement of the anisotropic core/shell particles leads to the formation of more ellipsoid diffusion zones of slightly differing sizes. Consequently, diffusion zones contain different amounts of carbon atoms and nucleation sites. Therefore, synthesized nanodiamonds provide slightly different sizes leading to a nanodiamonds size distribution. Thus, nanodiamond mean sizes and size distribution depend on the TNT shell thickness and on the level of anisotropy within the precursor structure.

(Figure 13). Therefore, the nanodiamond size depend only on the RDX mass fraction and the TNT shell thickness in the ideal case. Following the suggested mechanism for the ideal case it becomes obvious that the correlation between precursor particle size and nanodiamond size 
proposed by Pichot et al. remains untouched since it is not germane for estimating the nanodiamond size if the TNT carbon atoms which form the nanodiamonds arises from a single TNT nanoparticle or of four fourth RDX/TNT core/shell nanoparticles. However, under real conditions the hexolite precursor material consist of anisotropic core/shell particles exhibiting a size distribution. Due to the size distribution, a perfect symmetrical hexagonal close packing is not accomplishable even if a dense packing of the hexolite precursor material is undertaken before detonation synthesis (see Experimental section). Moreover, the anisotropy of the core/shell precursor particles (by meaning of the RDX core is not centered in the middle of the particle) causes a further decrease of the symmetry within the precursor structure. The symmetry decrease leads to formation of diffusion zones respectively diffusion barriers with more ellipsoid character and different dimensions (Figure 13). As a consequence, the thermodynamic and kinetic circumstances as well as the amount of TNT carbon atoms within the single diffusion zones differ leading to a varying number of nucleation sites. This results in slightly different nanodiamond sizes and thus in a nanodiamond size distribution. Consequently, the nanodiamond mean size $d_{n d}$ of the obtained nanodiamonds depends not only on the RDX mass fraction $\omega_{R D X}$ and the TNT shell thickness $d_{T N T}$ but also on the parameters defining the symmetry of the precursor material. These parameters are the hexolite precursor particle mean size $d_{\text {hex }}$, the hexolite precursor size distribution $s_{\text {hex }}$ and the level of anisotropy $a_{\text {hex }}$ within the precursor structure. Thus $d_{n d}$ can be assumed by the following correlation:

$$
d_{n d} \sim \omega_{R D X} d_{T N T}+d_{T N T}\left(a_{\text {hex }}+\frac{\left|s_{\text {hex }}\right|}{d_{\text {hex }}}\right)
$$

The level of anisotropy is defined as the distance between the hexolite particle center and the center of the corresponding RDX core $d_{\text {hex } \rightarrow R D X}$ divided by the hexolite particle mean radius $r_{\text {hex }}$ :

$$
a_{\text {hex }}=\frac{d_{\text {hex } \rightarrow R D X}}{r_{\text {hex }}}
$$

Equation 2

The described correlation presented in Equation 1 allows an interpretation of the nanodiamond mean sizes and size distributions of the presented detonation synthesized nanodiamonds. ND-80/20 provides with $6.1 \mathrm{~nm} \pm 0.2 \mathrm{~nm}$ the largest particle means size and the broadest size distribution. Due to the formation of patchy or Janus particles, the level of anisotropy $a_{\text {hex }}$ of 
SFE-80/20 nanoparticles has the highest value of all investigated hexolite nano-mixtures because $d_{\text {hex } \rightarrow R D X}$ is nearly equal to $r_{\text {hex }}$ (compare Figure 9.a). Furthermore $d_{T N T}$ - which is in case of SFE-80/20 more a TNT patch diameter than a shell thickness - is supposed to be relatively large since TNT solely form patches at single spots at the RDX surface. Additionally, the formation of patchy particles causes a reduction of the symmetry within the precursor charge compared to core/shell particles. Moreover, SFE-80/20 provides the broadest size distribution reducing further the symmetry of the precursor charge. Finally, SFE-80/20 contains the highest RDX mass fraction promoting higher temperatures and pressures inside the diffusion zones leading to a more complete conversation of carbon atoms to nanodiamonds and thus to lager nanodiamond mean sizes. Contrary to ND-80/20, nanodiamonds formed from RDX/TNT core/shell nanoparticles (ND-60/40, ND-40/60, ND-20/80) exhibit significant smaller particle mean sizes and narrower size distributions. ND-60/40 shows with $4.2 \mathrm{~nm} \pm 0.05 \mathrm{~nm}$ the largest particle mean size of all nanodiamonds synthesized from core/shell nanoparticles although the SFE-60/40 hexolite nanoparticles provide a relatively small mean size and TNT shell thickness. However, as already discussed at the building mechanism section above, RDX nucleates earlier the higher the RDX mass fractions. Consequently, RDX cores are more affected by the fictitious forces leading to a complete or nearly complete migration of the primary RDX nanoparticles towards the acetone droplet edges before TNT nucleation starts. As a consequence, SFE-60/40 shows a higher level of anisotropy $a_{\text {hex }}$ than SFE-40/60 and SFE-20/80 and thus a higher reduction of the precursor symmetry. Additionally, the relatively high RDX mass fraction supports the carbon atom to diamond conversion leading to larger nanodiamond mean sizes as discussed above. On the other hand, ND-20/80 exhibit a mean size of $3.7 \mathrm{~nm} \pm 0.08 \mathrm{~nm}$ though the SFE-20/80 precursor shows a larger core/shell nanoparticle means size and a larger TNT shell thickness $d_{T N T}$. Since this hexolite mixture includes the lowest RDX mass fraction SFE-20/80 particles should provide the smallest level of anisotropy of all presented hexolite mixtures. The lowest RDX mass fraction results further in the lowest temperature and pressure values inside the diffusion zones of all hexolite materials. Consequently, a less amount of carbon atoms converts into nanodiamonds leading to smaller nanodiamond mean sizes. Due to the smaller mean size of ND-20/80 compared to ND-60/40, the symmetry parameters, especially the level of anisotropy $a_{h e x}$, seem to affect the nanodiamond mean size at least as strong as the TNT shell thickness $d_{T N T}$ and the RDX mass fraction $\omega_{R D X}$. Anyway, the smallest nanodiamond mean size including the narrowest size distribution could be found at SFE-40/60-nd with $3.6 \pm 0.04 \mathrm{~nm}$. The SFE-40/60 hexolite precursor presents a thicker TNT shell but a less level of anisotropy $a_{\text {hex }}$ than SFE-60/40. Contrary, SFE-40/60 is defined by a 
thinner TNT shell but a higher level of anisotropy than SFE-20/80. Since the level of anisotropy $a_{\text {hex }}$ and the TNT shell thickness $d_{T N T}$ within an anisotropic hexolite core/shell nanoparticle should be as small as possible in order to receive the smallest nanodiamonds (Equation 1) but both parameters behave inversely proportional to each other (due to the varying RDX mass fractions), SFE-40/60 seems to provide the best mixture between its TNT shell thickness $d_{T N T}$ and its level of anisotropy $a_{\text {hex }}$.

\section{Conclusion}

Different hexolite mixtures with various RDX/TNT mass ratios were produced via Spray Flash Evaporation and investigated with AFM, confocal far-field Raman spectroscopy and TERS. The combination of these techniques gave insight into the sizes of the produced hexolite particles and into the arrangements of RDX and TNT molecules within single particles on the nanoscale. It was demonstrated that SFE-80(RDX)/20(TNT) is built up by patchy RDX/TNT nanoparticles while SFE-60/40, SFE-40/60 and SFE-20/80 form anisotropic RDX/TNT core/shell nanoparticles. The anisotropy of the particles, which depends on the RDX/TNT mass ratio, expresses a non-centered RDX core appearing closer to the hexolite nanoparticle edges. A building mechanism for the different SFE produced hexolite mixtures based on the experimental results and the dynamics of the SFE process is proposed. Due to the higher solubility, RDX nanoparticles are formed first during SFE process. Driven by fictitious forces, RDX nanoparticles migrate towards acetone droplet edges while TNT grows onto RDX nanoparticle surfaces. Since RDX nanoparticles are not centered in the droplet, hexolite nanoparticle formation occur anisotropic.

The influence of the hexolite precursor structure on the size and the distribution of the resulting nanodiamonds was discussed in detail. It is proposed that the TNT shell thickness, the symmetry reduction by meaning of the level of anisotropy (position of the RDX core center related to the hexolite particle center) and the precursor size distribution determine the resulting nanodiamond mean size. Further studies are necessary to verify the correlation between these parameters and the nanodiamond mean size. For this purpose, more RDX/TNT mass ratios have to be investigated to find the perfect relationship between TNT shell thickness and the anisotropy level within the RDX/TNT core/shell nanoparticles. This way, the size distribution of nanodiamonds produced from the detonation of hexolite mixtures could be further reduced. 


\section{Acknowledgement}

The authors thank Laurent Schlur for recording the presented SEM images. The authors also thank the Direction Générale de l'Armement (DGA, France) and the Bundesamt für Ausrüstung, Informationstechnik und Nutzung der Bundeswehr (BAAINBw, Germany). ISL is a joint initiative of the Ministère de la Défense (France) and the Bundesministerium der Verteidigung (Germany) and is actively supporting the NS3E joint laboratory including the Centre National de la Recherche Scientifique (CNRS) and the University of Strasbourg (UNISTRA). VD acknowledges support via the German Science Foundation (DFG) SFB 1278 Polytarget (Project B04).

\section{References}

1. Mochalin, V. N.; Shenderova, O.; Ho, D.; Gogotsi, Y., The properties and applications of nanodiamonds. Nature Nanotechnology 2012, 7 (1), 11-23.

2. Danilenko, V. V., On the history of the discovery of nanodiamond synthesis. Phys. Solid State 2004, 46.

3. Chang, Y. R., Mass production and dynamic imaging of fluorescent nanodiamonds. Nature Nanotech. 2008, 3.

4. Mochalin, V. N.; Gogotsi, Y., Wet chemistry route to hydrophobic blue fluorescent nanodiamond. J. Am. Chem. Soc. 2009, 131.

5. Shimkunas, R. A., Nanodiamond-insulin complexes as $\mathrm{pH}$-dependent protein delivery vehicles. Biomaterials 2009, 30.

6. Purtov, K. V.; Petunin, A. I.; Burov, A. E.; Puzyr, A. P.; Bondar, V. S., Nanodiamonds as carriers for address delivery of biologically active substances. Nanoscale Res. Lett. 2010, 5. 7. Alhaddad, A., Nanodiamond as a vector for siRNA delivery to Ewing sarcoma cells. Small 2011, 7.

8. Chow, E. K., Nanodiamond therapeutic delivery agents mediate enhanced chemoresistant tumor treatment. Sci. Transl. Med. 2011, 3.

9. Maze, J. R., Nanoscale magnetic sensing with an individual electronic spin in diamond. Nature 2008, 455.

10. Holt, K. B., Diamond at the nanoscale: Applications of diamond nanoparticles from cellular biomarkers to quantum computing. Phil. Trans. Roy. Soc. A 2007, 365.

11. Ivanov, M. G.; Pavlyshko, S. V.; Ivanov, D. M.; Petrov, I.; Shenderova, O., Synergistic compositions of colloidal nanodiamond as lubricant-additive. J Vac. Sci. Technol. B 2010, 28. 
12. Dolmatov, V. Y., Detonation synthesis ultradispersed diamonds: Properties and applications. Usp. Khim. 2001, 70.

13. Guillevic, M.; Pichot, V.; Fioux, P.; Schnell, F.; Spitzer, D., Nanodiamond-based energetic core-shell composites: The route towards safer materials. Diamond Relat. Mater. 2019, $93,150-158$.

14. Danilenko, V. V., Synthesis, Properties and Applications of Ultrananocrystalline Diamond. 2005.

15. Shenderova, O. A.; Gruen, D. M., Ultrananocrystalline Diamond: Synthesis, Properties, and Applications. 2006.

16. Anischkin, V. F., Isotope Studies of Detonation Mechanisms of TNT, RDX and HMX. Combustion, Explosion and Shock Waves 2007, 43.

17. Kozyrev, N. V.; Golubeva, E. S., Investigation of the synthesis of ultradispersed diamonds in mixtures of TNT with RDX, HMX and PETN. Combustion, Explosion and Shock Waves 1992, 28.

18. Malkov, I. Y. u., Formation of diamond from the liquid phase carbon. Combustion, Explosion and Shock Waves 1993, 29.

19. Titov, V. M.; Tolochko, B. P.; Ten, K. A.; Lukyanchikov, L. A.; Zubkov, P. I., The formation kinetics of detonation nanodiamonds. In Synthesis, Properties and Applications of Ultrananocrystalline Diamond, Springer: 2005; pp 169-180.

20. Titov, V. M.; Anisichkin, V. F.; Mal'kov, I. Y. u., Synthesis of ultradispersed diamond in detonation waves. Combustion, Explosion and Shock Waves 1989, 25.

21. Titov, V. M.; Tolochko, B. P.; Ten, K. A.; Lukyanchikov, L. A.; Pruuel, E. R., Where and when are nanodiaomnds formed under explosion? Diam. Relat. Mater. 2007, 16.

22. Chernishev, A. P., Physical-chemical model of nanodiamond formation at explosion. Nuclear Instruments and Methods in Physics Research A 2004, 575.

23. Pichot, V.; Comet, M.; Risse, B.; Spitzer, D., Detonation of Nanosized Explosive: New Mechanistic Model for Nanodiamond Formation. Diamond Relat. Mater. 2015, 54, 59-63.

24. Pichot, V.; Risse, B.; Schnell, F.; Mory, J.; Spitzer, D., Understanding ultrafine nanodiamond formation using nanostructured explosives. Sci. Rep. 2013, 3 (1), 2159.

25. Risse, B.; Hassler, D.; Spitzer, D. Preparation of Nanoparticles by Flash Evaporation. US20150000846A1, 2015.

26. Risse, B.; Spitzer, D.; Hassler, D.; Schnell, F.; Comet, M.; Pichot, V.; Muhr, H., Continuous Formation of Submicron Energetic Particles by the Flash-Evaporation Technique. Chem. Eng. J. 2012, 203, 158-165. 
27. Risse, B.; Schnell, F.; Spitzer, D., Synthesis and Desensitization of Nano- $\beta$-HMX. Propellants Explos. Pyrotech. 2014, 39 (3), 397-401.

28. Spitzer, D.; Pichot, V.; Risse, B. Method for manufacturing nanoparticles by detonation. US20150157997A1, 2013.

29. Spitzer, D.; Risse, B.; Schnell, F.; Pichot, V.; Klaumünzer, M.; Schaefer, M., Continuous Engineering of Nano-Cocrystals for Medical and Energetic Applications. Sci. Rep. 2014, 4.

30. Blas, L.; Klaumünzer, M.; Pessina, F.; Braun, S.; Spitzer, D., Nanostructuring of Pure and Composite-Based K6 Formulations with Low Sensitivities. Propellants Explos. Pyrotech. 2015, 40 (6), 938-944.

31. Hübner, J.; Pichot, V.; Guillevic, M.; Spitzer, D., Structure Investigation of Energetic Nanocomposites Produced by Spray Flash Evaporation via AFM-TERS. In ICORS, South Korea, 2018.

32. Hübner, J.; Deckert-Gaudig, T.; Glorian, J.; Deckert, V. K.; Spitzer, D., Surface Characterization of Nanoscale Co-Crystals Enabled through Tip Enhanced Raman Spectroscopy. Nanoscale 2020, DOI: 10.1039/D0NR00397B.

33. Hübner, J.; Klaumünzer, M.; Comet, M.; Martin, C.; Vidal, L.; Schäfer, M.; Kryschi, C.; Spitzer, D., Insights into Combustion Mechanisms of Variable Aluminum-Based Iron Oxide/-Hydroxide Nanothermites. Combust. Flame 2017, 184, 186-194.

34. Trautmann, S.; Aizpurua, J.; Götz, I.; Undisz, A.; Dellith, J.; Schneidewind, H.; Rettenmayr, M.; Deckert, V., A Classical Description of Subnanometer Resolution by Atomic Features in Metallic Structures. Nanoscale 2017, 9 (1), 391-401.

35. Deckert-Gaudig, T.; Taguchi, A.; Kawata, S.; Deckert, V., Tip-Enhanced Raman Spectroscopy-From Early Developments to Recent Advances. Chem. Soc. Rev. 2017.

36. Roth, R. M.; Panoiu, N. C.; Adams, M. M.; Osgood, R. M.; Neacsu, C. C.; Raschke, M. B., Resonant-Plasmon Field Enhancement from Asymmetrically Illuminated Conical MetallicProbe Tips. Opt. Express 2006, 14 (7), 2921-2931.

37. Pettinger, B.; Domke, K. F.; Zhang, D.; Schuster, R.; Ertl, G., Direct Monitoring of Plasmon Resonances in a Tip-Surface Gap of Varying Width. Phys. Rev. B: Condens. Matter 2007, 76 (11), 113409.

38. Watanabe, H.; Ishida, Y.; Hayazawa, N.; Inouye, Y.; Kawata, S., Tip-Enhanced NearField Raman Analysis of Tip-Pressurized Adenine Molecule. Phys. Rev. B: Condens. Matter 2004, 69 (15), 155418-1-155418-11. 
39. Ichimura, T.; Watanabe, H.; Morita, Y.; Verma, P.; Kawata, S.; Inouye, Y., Temporal Fluctuation of Tip-Enhanced Raman Spectra of Adenine Molecules. J. Phys. Chem. C 2007, 111 (26), 9460-9464.

40. Deckert-Gaudig, T.; Rauls, E.; Deckert, V., Aromatic Amino Acid Monolayers Sandwiched Between Gold and Silver: A Combined Tip-Enhanced Raman and Theoretical Approach. J. Phys. Chem. C 2009, 114 (16), 7412-7420.

41. Sonntag, M. D.; Klingsporn, J. M.; Garibay, L. K.; Roberts, J. M.; Dieringer, J. A.; Seideman, T.; Scheidt, K. A.; Jensen, L.; Schatz, G. C.; Van Duyne, R. P., Single-molecule tipenhanced Raman spectroscopy. J. Phys. Chem. C 2011, 116 (1), 478-483.

42. Klingsporn, J. M.; Jiang, N.; Pozzi, E. A.; Sonntag, M. D.; Chulhai, D.; Seideman, T.; Jensen, L.; Hersam, M. C.; Duyne, R. P. V., Intramolecular Insight Into Adsorbate-Substrate Interactions via Low-Temperature, Ultrahigh-Vacuum Tip-Enhanced Raman Spectroscopy. $J$. Am. Chem. Soc. 2014, 136 (10), 3881-3887.

43. Bailo, E.; Deckert, V., Tip-Enhanced Raman Spectroscopy of Single RNA Strands: Towards a Novel Direct-Sequencing Method. Angew. Chem. Int. Ed. 2008, 47 (9), 1658-1661. 44. Deckert-Gaudig, T.; Pichot, V.; Spitzer, D.; Deckert, V., High-Resolution Raman Spectroscopy for the Nanostructural Characterization of Explosive Nanodiamond Precursors. ChemPhysChem 2017, 18 (2), 175-178.

45. Infante-Castillo, R.; Pacheco-Londoño, L.; Hernández-Rivera, S. P., Vibrational Spectra and Structure of RDX and its 13C-and 15N-Labeled Derivatives: A Theoretical and Experimental Study. Spectrochim. Acta A 2010, 76 (2), 137-141.

46. Miao, M. S.; Dreger, Z. A.; Winey, J. M.; Gupta, Y. M., Density Functional Theory Calculations of Pressure Effects on the Vibrational Structure of $\alpha$-RDX. The Journal of Physical Chemistry A 2008, 112 (47), 12228-12234.

47. Dreger, Z. A.; Gupta, Y. M., High Pressure Raman Spectroscopy of Single Crystals of Hexahydro-1,3,5-trinitro-1,3,5-triazine (RDX). The Journal of Physical Chemistry B 2007, 111 (15), 3893-3903.

48. Liu, Y.; Perkins, R.; Liu, Y.; Tzeng, N., Normal Mode and Experimental Analysis of TNT Raman Spectrum. J. Mol. Struct. 2017, 1133, 217-225.

49. Clarkson, J.; Smith, W. E.; Batchelder, D. N.; Smith, D. A.; Coats, A. M., A Theoretical Study of the Structure and Vibrations of 2, 4, 6-Trinitrotolune. J. Mol. Struct. 2003, 648 (3), 203-214.

50. Bailo, E.; Deckert, V., Tip-enhanced Raman spectroscopy of single RNA strands: towards a novel direct-sequencing method. Angew. Chem. Int. Ed. 2008, 47 (9), 1658-1661. 
51. Goetz, F.; Brill, T., Laser Raman Spectra of Alpha.-, Beta.-, Gamma.-, and Deltaoctahydro-1, 3, 5, 7-tetranitro-1, 3, 5, 7-tetrazocine and Their Temperature Dependence. $J$. Phys. Chem. 1979, 83 (3), 340-346.

52. Goede, P.; Latypov, N. V.; Östmark, H., Fourier Transform Raman Spectroscopy of the Four Crystallographic Phases of $\alpha, \beta, \gamma$ and $\varepsilon 2,4,6,8,10,12$-Hexanitro-2, 4, 6, 8, 10, 12 hexaazatetracyclo-[5.5. 0.05, 9.03, 11]-dodecane (HNIW, CL-20). Propellants Explos. Pyrotech. 2004, 29 (4), 205-208.

53. Infante-Castillo, R.; Pacheco-Londoño, L. C.; Hernández-Rivera, S. P., Monitoring the $\alpha \rightarrow \beta$ Solid-Solid Phase Transition of RDX with Raman Spectroscopy: A Theoretical and Experimental Study. J. Mol. Struct. 2010, 970 (1-3), 51-58.

54. Watanabe, H.; Hayazawa, N.; Inouye, Y.; Kawata, S., DFT Vibrational Calculations of Rhodamine 6G Adsorbed on Silver: Analysis of Tip-Enhanced Raman Spectroscopy. The Journal of Physical Chemistry B 2005, 109 (11), 5012-5020.

55. Stadler, J.; Schmid, T.; Zenobi, R., Nanoscale Chemical Imaging of Single-Layer Graphene. ACS Nano 2011, 5 (10), 8442-8448.

56. Opilik, L.; Payamyar, P.; Szczerbiński, J.; Schütz, A. P.; Servalli, M.; Hungerland, T.; Schlüter, A. D.; Zenobi, R., Minimally Invasive Characterization of Covalent Monolayer Sheets Using Tip-Enhanced Raman Spectroscopy. ACS Nano 2015, 9 (4), 4252-4259.

57. Karthika, S.; Radhakrishnan, T. K.; Kalaichelvi, P., A Review of Classical and Nonclassical Nucleation Theories. Cryst. Growth Des. 2016, 16 (11), 6663-6681.

58. Oura, K.; Lifshits, V. G.; Saranin, A. A.; Zotov, A. V.; Katayama, M., Growth of Thin Films. In Surface Science: An Introduction, Springer: 2013; pp 357-387.

59. Chung, J. N., The Motion of Particles Inside a Droplet. J. Heat Transfer 1982, 104 (3), 438-445.

60. Ali, A. M.; Deckert-Gaudig, T.; Egiza, M.; Deckert, V.; Yoshitake, T., Near- and farfield Raman spectroscopic studies of nanodiamond composite films deposited by coaxial arc plasma. Appl. Phys. Lett. 2020, 116 (4), 041601. 
Formation Mechanism of Anisotropic RDXTNT CoreShell ... (2.51 MiB) view on ChemRxiv • download file 


\section{Formation Mechanism of Anisotropic RDX/TNT Core/Shell Nanoparticles and their Influence onto Nanodiamond Detonation Syntheses}

Jakob Hübner ${ }^{[\mathrm{a}]}$, Vincent Pichot ${ }^{[\mathrm{a}]}$, Emeline Lobry ${ }^{[\mathrm{a}]}$, Tanja Deckert-Gaudig ${ }^{[\mathrm{b}]}$, Volker Deckert $^{[\mathrm{b}, \mathrm{c}, \mathrm{d}]}$, Denis Spitzer ${ }^{[\mathrm{a}]}$

[a] Nanomatériaux pour les Systèmes Sous Sollicitations Extrêmes (NS3E), ISL-CNRSUNISTRA UMR 3208, French-German Research Institute of Saint-Louis, 5, rue du Général Cassagnou, B.P. 70034, 68301 Saint-Louis, France

[b] Leibniz Institute of Photonic Technology (IPHT), Albert-Einstein-Str. 9, 07745 Jena, Germany

[c] Institute of Physical Chemistry and Abbe Center of Photonics, Friedrich-Schiller-University Jena, Helmholtzweg 4, 07743 Jena, Germany

[d] Institute of Quantum Science and Engineering, Texas A\&M University, College Station, TX 77843-4242, USA

\section{Corresponding Author}

Correspondence should be addressed to jakob.huebner@isl.eu 


\section{Supporting Information}

S1. Raman active vibrational frequencies and assignments

S2. Size distributions of SFE produced hexolite nanocomposites

S3. Measurement of droplet size and velocity in the SFE process

S4. Estimation of RDX core diameters and TNT shell thicknesses for isotropic hexolite core/shell nanoparticles 
S1. Raman active vibrational frequencies and assignments

\section{1,3,5-Trinitro-1,3,5-triazinane (RDX)}

In the following measured Raman active frequencies of pristine 1,3,5-Trinitro-1,3,5-triazinane (RDX) are presented, assigned and compared with literature.

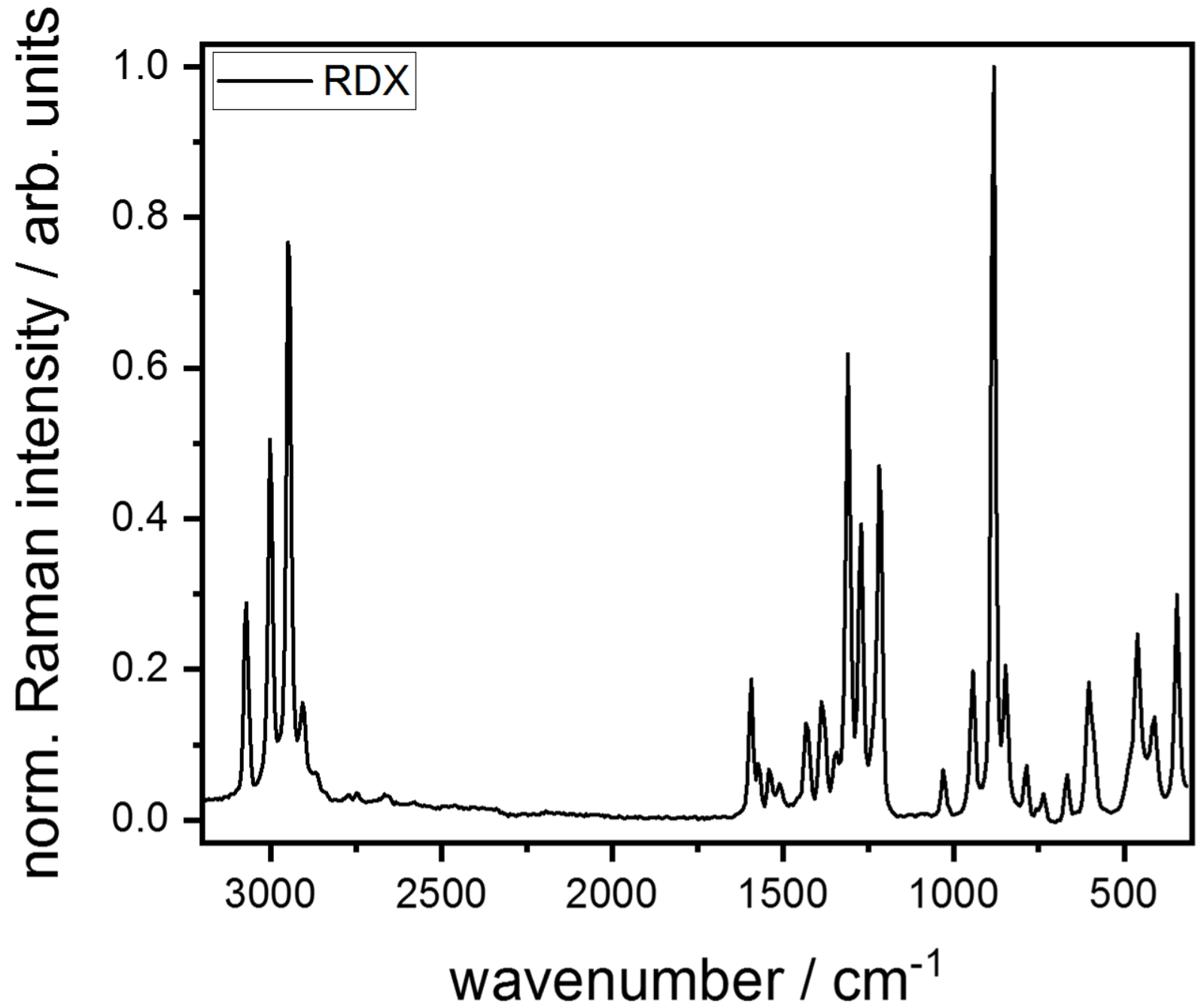

Figure 1. Raman spectrum of RDX in a spectral range between $3200 \mathrm{~cm}^{-1}$ and $300 \mathrm{~cm}^{-1}$. 
Table 1. Raman frequencies and their assignments of RDX

\begin{tabular}{|c|c|c|}
\hline \multicolumn{3}{|c|}{ Raman frequencies of $\mathrm{RDX}$} \\
\hline Experiment $/ \mathrm{cm}^{-1}$ & Literature / $\mathrm{cm}^{-1}[1]$ & Assignments [1-3] \\
\hline $345 \mathrm{~m}$ & 347 & ring twist \\
\hline $410 \mathrm{w}$ & 415 & ring bend \\
\hline $461 \mathrm{~m}$ & 464 & ring bend, $\mathrm{N}-\mathrm{N}$ str \\
\hline $489 \mathrm{vw}$ & 488 & ring twist, $\mathrm{NO}_{2}$ sci \\
\hline $589 \mathrm{w}$ & 588 & ring twist \\
\hline $603 \mathrm{w}$ & 607 & ring rock \\
\hline $667 \mathrm{vw}$ & 670 & ring bend \\
\hline $736 \mathrm{vw}$ & 739 & $\mathrm{~N}-\mathrm{NO}_{2} \mathrm{umb}$ \\
\hline $757 \mathrm{vw}$ & 757 & ring bend, $\mathrm{NO}_{2}$ sci \\
\hline $785 \mathrm{vw}$ & 788 & $\mathrm{C}-\mathrm{N}$ str, $\mathrm{NO}_{2}$ sci \\
\hline $847 \mathrm{~m}$ & 848 & $\mathrm{~N}-\mathrm{N}$ str, $\mathrm{NO}_{2}$ sci \\
\hline 882 vs & 885 & ring breath \\
\hline $943 \mathrm{w}$ & 945 & $\mathrm{~N}-\mathrm{N}$ str \\
\hline $1024 \mathrm{vw}$ & 1023 & $\mathrm{CH}_{2}$ rock \\
\hline $1031 \mathrm{vw}$ & 1031 & $\mathrm{~N}-\mathrm{C}$ str \\
\hline $1218 \mathrm{~m}$ & 1215 & $\mathrm{~N}-\mathrm{C}$ str \\
\hline $1270 \mathrm{w}$ & 1273 & $\mathrm{~N}-\mathrm{NO}_{2}$ str sym \\
\hline $1309 \mathrm{~s}$ & 1309 & $\mathrm{CH}_{2}$ twist, $\mathrm{N}-\mathrm{NO}_{2}$ str \\
\hline $1346 \mathrm{vw}$ & 1346 & $\mathrm{CH}_{2}$ wag \\
\hline $1377 \mathrm{w}$ & 1377 & $\mathrm{CH}_{2}$ twist \\
\hline $1387 \mathrm{w}$ & 1388 & $\mathrm{CH}_{2}$ wag \\
\hline $1432 \mathrm{w}$ & 1436 & $\mathrm{CH}_{2}$ sci \\
\hline $1458 \mathrm{vw}$ & 1460 & $\mathrm{CH}_{2} \mathrm{sci}$ \\
\hline $1509 \mathrm{vw}$ & 1508 & $\mathrm{CH}_{2} \mathrm{sci}$ \\
\hline $1541 \mathrm{vw}$ & 1542 & $\mathrm{NO}_{2}$ str asym \\
\hline $1573 \mathrm{vw}$ & 1573 & $\mathrm{NO}_{2}$ str asym \\
\hline $1592 \mathrm{w}$ & 1595 & $\mathrm{NO}_{2}$ str asym \\
\hline $2907 \mathrm{w}$ & 2906 & C-H str \\
\hline 2949 vs & 2949 & C-H str \\
\hline $3003 \mathrm{~s}$ & 3003 & C-H str \\
\hline $3072 \mathrm{~m}$ & 3067 & C-H str \\
\hline $3077 \mathrm{~m}$ & 3075 & C-H str \\
\hline
\end{tabular}

RDX marker band vibration $\left(882 \mathrm{~cm}^{-1}\right.$; ring breathing) is marked in green. Notes: vs - very strong, $\mathrm{s}$ - strong, $\mathrm{m}$ - medium, w - weak, vw - very weak; asym - asymmetric, bend - bending, breath - breathing, rock - rocking, sci - scissoring, sym - symmetric, , str - stretching, twist twisting, umb - umbrella, wag - wagging 


\section{2-Methyl-1,3,5-trinitrobenzene (TNT)}

In the following measured Raman active frequencies of pristine 2-Methyl-1,3,5-trinitrobenzene (TNT) are summed up, assigned and compared with literature.

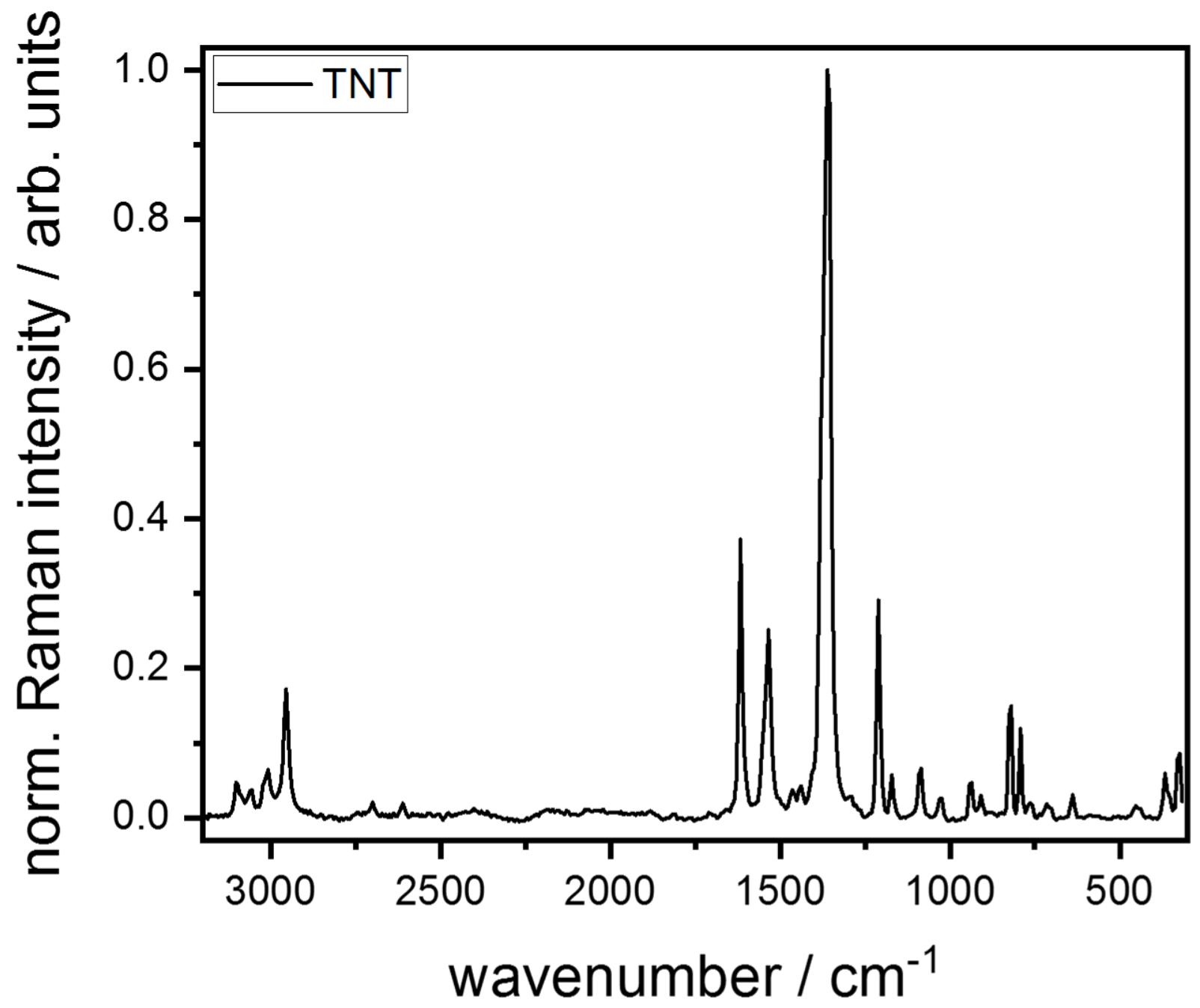

Figure 2. Raman spectrum of RDX in a spectral range between $3200 \mathrm{~cm}^{-1}$ and $300 \mathrm{~cm}^{-1}$.

Table 2. Raman frequencies and their assignments of TNT

\begin{tabular}{|l|l|l|}
\hline \multicolumn{3}{|c|}{ Raman frequencies of TNT } \\
\hline Experiment $/ \mathbf{c m}^{-1}$ & Literature $/ \mathbf{c m}^{-1}[\mathbf{4}]$ & Assignments $[3, \mathbf{4}]$ \\
\hline $323 \mathrm{w}$ & 326 & C-N tors, ring bend \\
\hline $352 \mathrm{vw}$ & 355 & ring bend \\
\hline $367 \mathrm{w}$ & 366 & $\mathrm{CH}_{3}$ def, ring bend \\
\hline $439 \mathrm{vw}$ & 442 & $\mathrm{C}-\mathrm{CH}_{3}$ wag \\
\hline $453 \mathrm{vw}$ & 457 & ring tors \\
\hline $638 \mathrm{vw}$ & 639 & ring bend \\
\hline $701 \mathrm{vw}$ & 704 & ring tors \\
\hline
\end{tabular}




\begin{tabular}{|c|c|c|}
\hline $716 \mathrm{vw}$ & 717 & $4 \mathrm{NO}_{2}$ bend \\
\hline $736 \mathrm{vw}$ & 736 & ring bend, 2,6- $\mathrm{NO}_{2}$ sci \\
\hline $764 \mathrm{vw}$ & 760 & $2,4,6 \mathrm{NO}_{2}$ bend, ring tors \\
\hline $792 \mathrm{~m}$ & 792 & $\begin{array}{l}\text { ring bend, } \mathrm{C}-\mathrm{CH}_{3} \text { str, } 2,4,6- \\
\mathrm{NO}_{2} \text { sci }\end{array}$ \\
\hline $819 \mathrm{~m}$ & 822 & $2,4,6 \mathrm{NO}_{2}$ sci, ring bend \\
\hline $908 \mathrm{vw}$ & 907 & $\begin{array}{l}2,4,6 \mathrm{NO}_{2} \text { sci, ring bend, } \\
\text { C-N str }\end{array}$ \\
\hline $936 \mathrm{w}$ & 939 & $\mathrm{C}-\mathrm{H}$ (ring) bend \\
\hline $1024 \mathrm{vw}$ & 1025 & $\mathrm{CH}_{3}$ def \\
\hline $1085 \mathrm{w}$ & 1086 & C-H (ring) bend \\
\hline $1171 \mathrm{w}$ & 1170 & $\begin{array}{l}\text { C-C (ring) bend, 2,4,6 C-N } \\
\text { str, C-CH } \mathrm{CH}_{3} \text { str }\end{array}$ \\
\hline $1211 \mathrm{~s}$ & 1210 & ring breath \\
\hline $1290 \mathrm{vw}$ & 1292 & ring $\mathrm{str}$ \\
\hline $1358 \mathrm{vs}$ & 1359 & $4 \mathrm{NO}_{2}$ sym str, C-N str \\
\hline $1365 \mathrm{vs}$ & 1365 & $2,6 \mathrm{NO}_{2}$ sym str, C-N str \\
\hline $1371 \mathrm{vs}$ & 1375 & $2,4,6 \mathrm{NO}_{2}$ sym str, $\mathrm{C}-\mathrm{N}$ str \\
\hline $1383 \mathrm{~s}$ & 1381 & $\mathrm{CH}_{3} \mathrm{umb}$ \\
\hline $1407 \mathrm{w}$ & 1403 & ring str \\
\hline $1438 \mathrm{vw}$ & 1438 & ring str, $\mathrm{CH}_{3}$ def \\
\hline $1445 \mathrm{vw}$ & 1446 & $\mathrm{CH}_{3}$ def \\
\hline $1465 \mathrm{vw}$ & 1466 & $\mathrm{CH}_{3}$ def \\
\hline $1534 \mathrm{~s}$ & 1533 & $4 \mathrm{NO}_{2}$ asym str, ring str \\
\hline $1553 \mathrm{~m}$ & 1551 & $2,4,6 \mathrm{NO}_{2}$ asym str \\
\hline $1617 \mathrm{~s}$ & 1618 & 2,6 asy str, ring str \\
\hline $2955 \mathrm{~m}$ & - & $\mathrm{C}-\mathrm{H}$ (methyl) str \\
\hline $3008 \mathrm{w}$ & - & C-H (ring) str \\
\hline $3056 \mathrm{vw}$ & - & C-H (ring) str \\
\hline 3103 vw & - & C-H (ring) str \\
\hline
\end{tabular}

TNT marker band vibrations $\left(1358 \mathrm{~cm}^{-1}-1371 \mathrm{~cm}^{-1}\right.$; three $\mathrm{NO}_{2}$ symmetric stretching vibrations) is marked in red. Notes: vs - very strong, s - strong, $\mathrm{m}$ - medium, w - weak, vw very weak; asym - asymmetric, bend - bending, breath - breathing, def - deformation, rock rocking, sci - scissoring, sym - symmetric, str - stretching, tors - torsion, twist - twisting, umb - umbrella, wag - wagging 


\section{S2. Size distributions of SFE-produced hexolite nano-composites}

Particle size distributions of all SFE produced hexolite nanocomposites were estimated by Atomic Force Microscopy. For this purpose the heights of 200 nanoparticles of each hexolite mixture were measured. The results, the fitted size distributions and the particle mean sizes $\left(d_{\text {hex }}\right)$ of each hexolite sample are presented in Figure 3. Figure 4.a) (and b) depicts an example AFM topographic map of SFE-40/60 nanoparticles which is typical for hexolite size distribution AFM measurements. Particles with relatively large heights were measured individually by using smaller scan areas and less scan rates to ensure if they consist of single nanoparticles or if they are single submicron particles. If these individual AFM scans could clearly identify agglomerates these particles were not counted. Contrary, if an agglomeration of single nanoparticles could not be proven, the particle was considered within the statistics. An AFM topographic map of one of these typical agglomerates (SFE-20/80) is depicted in Figure 4.c) (and d). The height profile shows unambiguously that the scanned agglomerate is formed by several single nanoparticles.
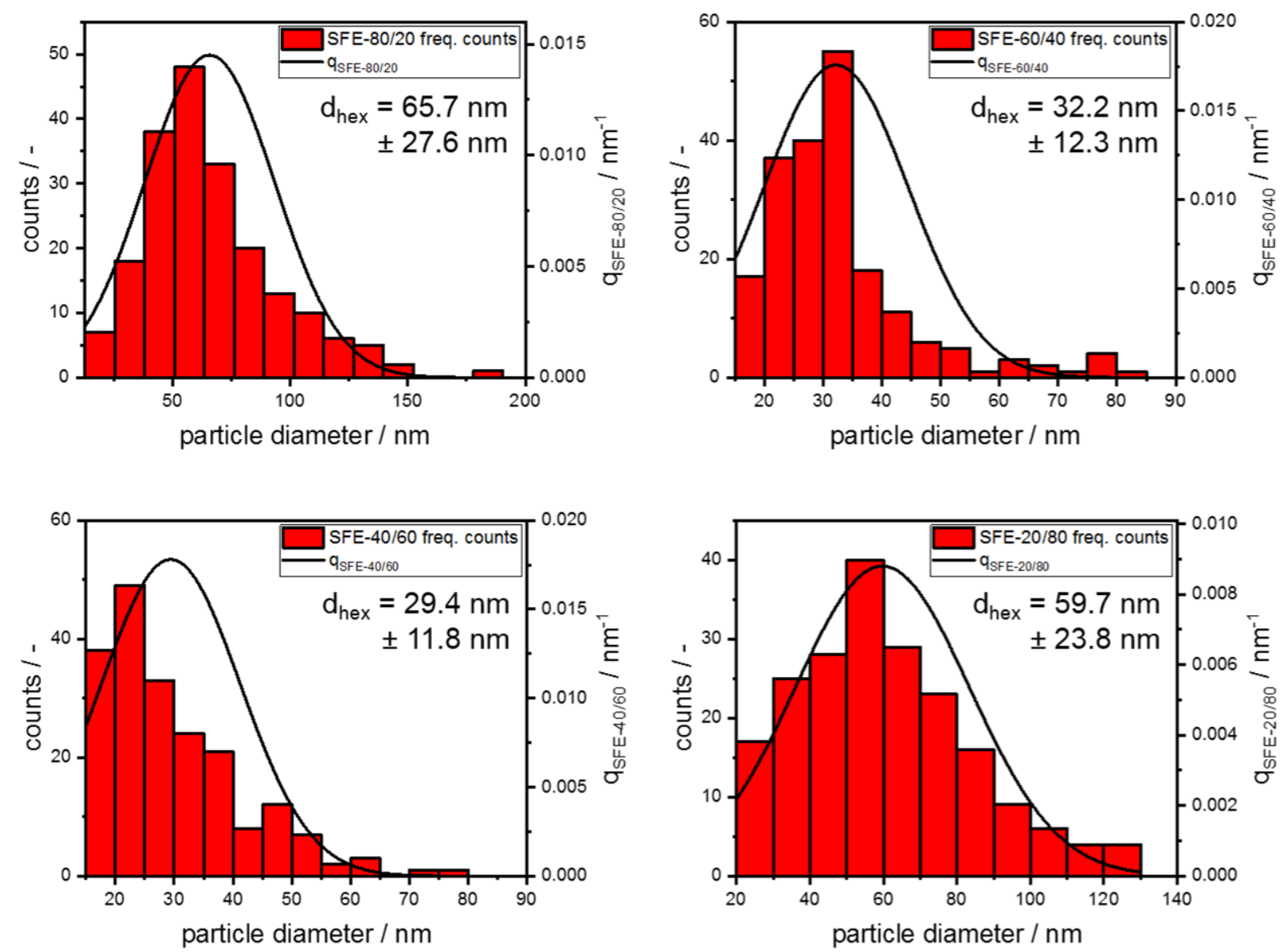

Figure 3. Size distribution of SFE-produced hexolite nanoparticles. 

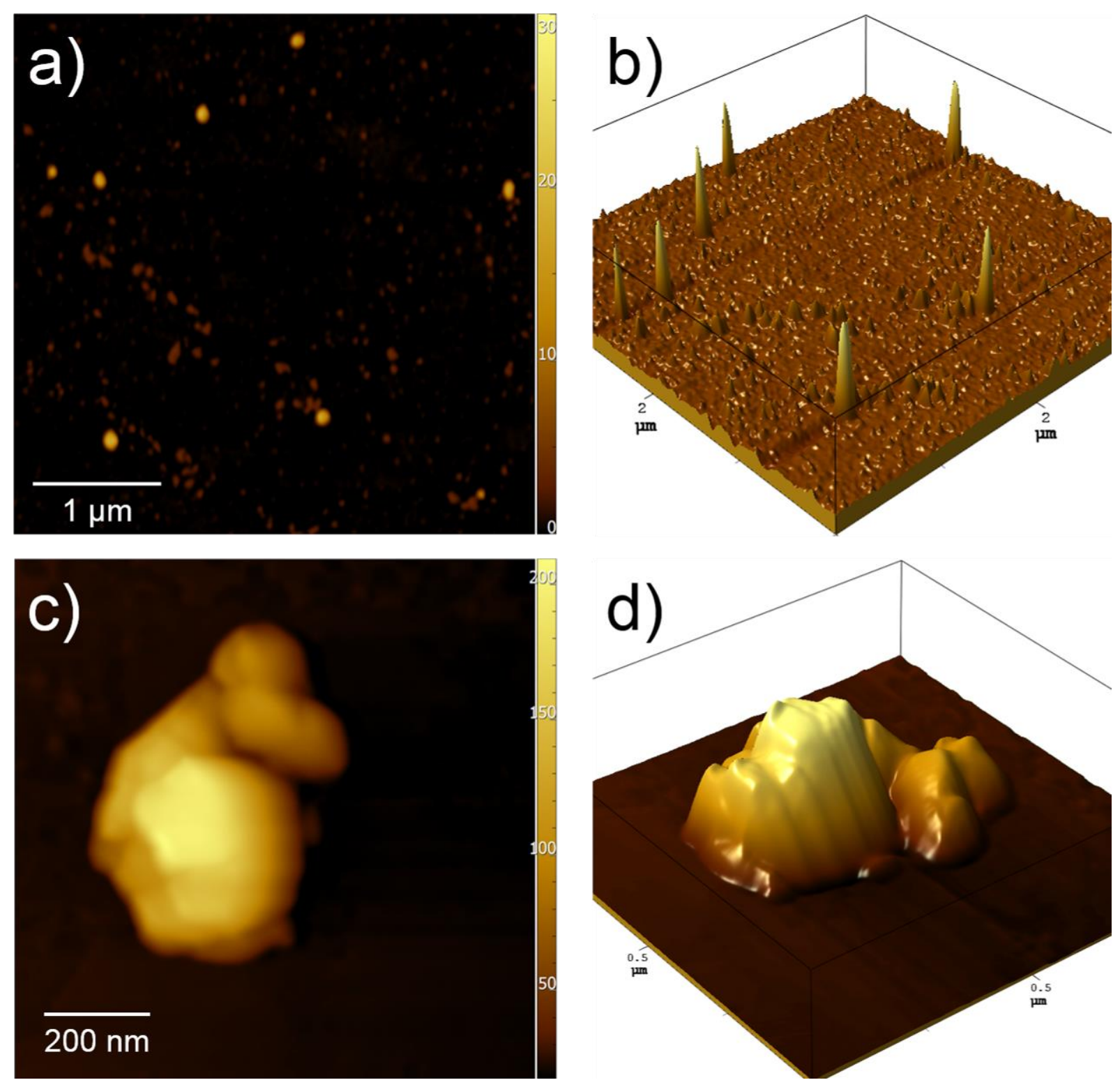

Figure 4. a) AFM topographic map of SFE-40/60. b) 3D representation of a). c) AFM topographic map of a SFE40/60 agglomerate. d) 3D representation of c). 


\section{S3. Measurement of droplet size and velocity in the SFE process}

In order to obtain information about the acetone droplets size evolution and their axial and radial velocities as functions of the distance from the nozzle during the Spray Flash Evaporation (SFE) process, Phase Doppler particle analysis (PDPA) measurements were performed on a pure acetone spray using the same SFE conditions as presented in the experimental section of the main text (nozzle diameter: $80 \mu \mathrm{m}$; nozzle temperature: $160{ }^{\circ} \mathrm{C}$; pressure: $40 \mathrm{bar}$ ). The used PDPA device consists of a FlowExplorer 2D (Dantec Dynamics, Danemark), a HiDense PDA receiver (Dantec Dynamics, Danemark) and a BSA P800 burst spectrum analyzer (Dantec Dynamics, Danemark).The FlowExplorer 2D act as light source and consists of two diode lasers with respective wavelengths of $532 \mathrm{~nm}$ and $561 \mathrm{~nm}$ and an output power of $300 \mathrm{~mW}$. These laser beams form an intersection point at a set focal length of $200 \mathrm{~mm}$ behind the light source. The intersection volume of the two lasers defines the measurable volume which is formed by an ellipsoid with a length of $0.68 \mathrm{~mm}$, a diameter of $0.1 \mathrm{~mm}$ and thus a resulting volume of $3.56 \cdot 10^{-3} \mathrm{~mm}^{3}$. Light scattered at acetone droplets is collected by the FiberPDA receiver which is equipped with three photodiodes allowing a large measurable size range and a high resolution. The receiver optics provide a focal length of $310 \mathrm{~mm}$ from the laser intersection. The angle $\varphi$ between light source and receiver optics is set to $120^{\circ}$. To enable spray investigations of different spray areas, the whole optical setup is installed on an adjustable 3D axis table. The axial droplet velocity $U(z-$ direction, Figure $5 . a)$ is measured by the $532 \mathrm{~nm}$ laser $\left(\lambda_{1}\right)$, the radial droplet velocity V (y - direction, Figure 5.a) is measured by the $561 \mathrm{~nm}$ laser $\left(\lambda_{2}\right)$. 20000 droplets were measured at each PDPA measurement. The axial velocity $U$ and the radial velocity $\mathrm{V}$ were calculated from the Doppler frequency $f_{D}$ with

$$
U=\frac{\lambda_{1}}{2 \sin (\theta / 2)} f_{D}
$$

Eq. 1

Respectively

$$
V=\frac{\lambda_{2}}{2 \sin (\theta / 2)} f_{D}
$$

where $\theta$ represents the angle between the two laser beams at the beam intersection. 
The droplet size $s_{d}$ was calculated by the phase difference $\Phi$ between the signals of two detectors with

$$
S_{D}=\frac{\Phi \lambda}{2 \pi} \cdot \frac{\sqrt{2(1-\cos \theta \cos \psi \cos \varphi}}{\sin \theta \sin \psi}
$$

where $\psi$ represents the angle between two scattered light beams receiving two different photo detectors at the laser beam intersection. Figure 5.a) and Figure 5.b) display the distribution of the axial respectively radial velocity as a function of the scanned areas. Figure $5 \mathrm{c}$ ) depicts the droplet mean size distribution in the spray as a function of the scanned area. The origin of the coordinate system was set to the nozzle position. Figure 6 gives the evolution of the droplet mean size, the axial velocity and the radial velocity as functions of the distance from the nozzle.

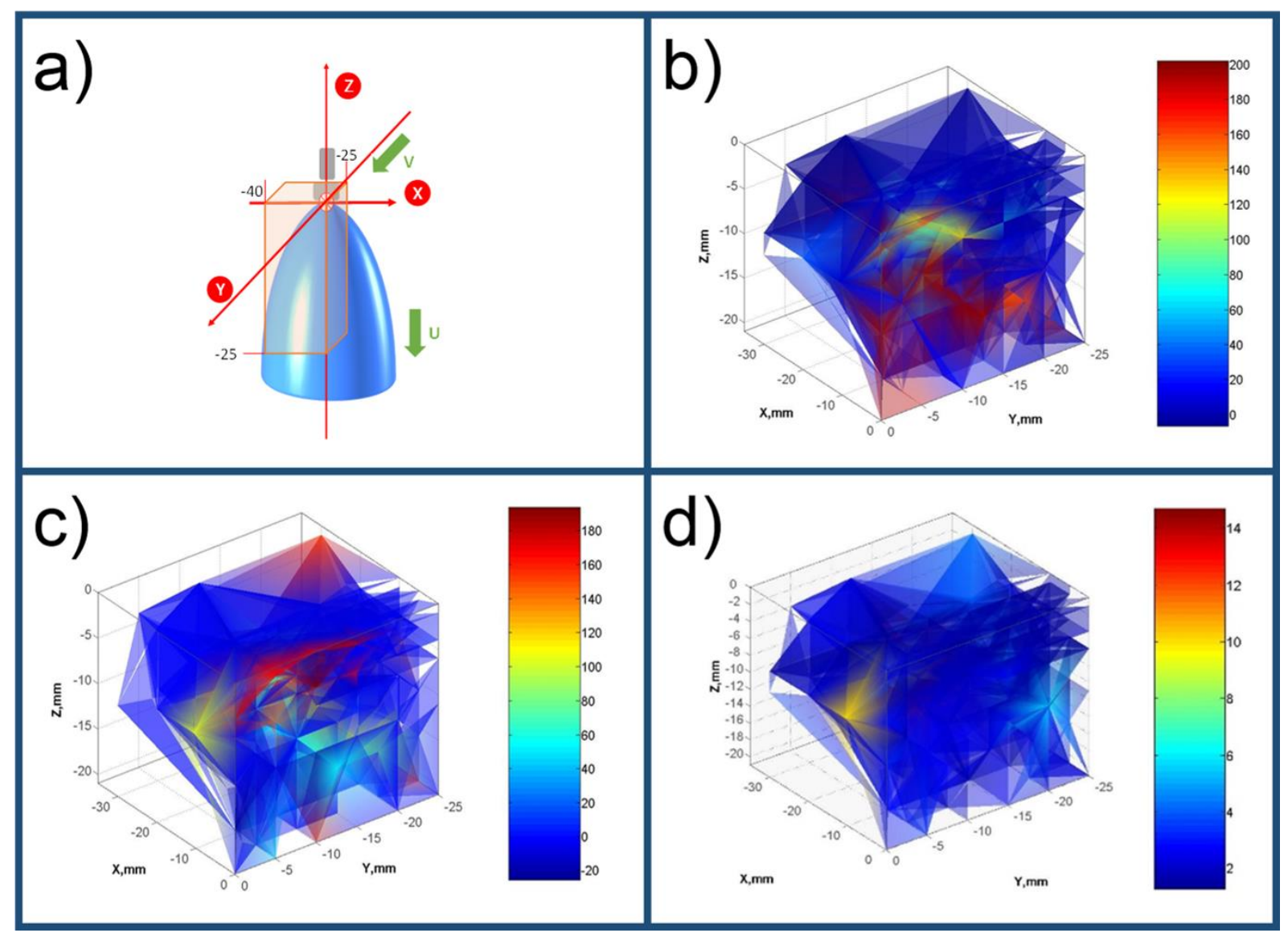

Figure 5. a) Schematic representation of the spray and the measurement zone. The measurement zone depends on $\mathrm{x}-, \mathrm{y}-$ and $\mathrm{z}-$ positions set by the $3 \mathrm{D}$ axes table. The origin of the coordinate system is centered at the nozzle outlet position. Shown dimensions are (given in $\mathrm{mm}$ ). b) Mean axial velocity $U$ distribution in the first quarter of the spray (given in $\mathrm{ms}^{-1}$ ). c) Mean radial velocity $\mathrm{V}$ distribution in the first quarter of the spray (given in $\mathrm{ms}^{-1}$ ). d) Mean droplet size distribution in the first quarter of the spray (given in $\mu \mathrm{m}$ ). 
Thus, the droplet mean size was found as $2.6 \mu \mathrm{m} \pm 0.8 \mu \mathrm{m}$ behind the nozzle. The shrinking of the droplet size follows approximately a linear decay. The partially negative velocity and the relatively large error bars of the radial velocity V (Figure 6, right) can be interpreted as turbulences inside the spray.
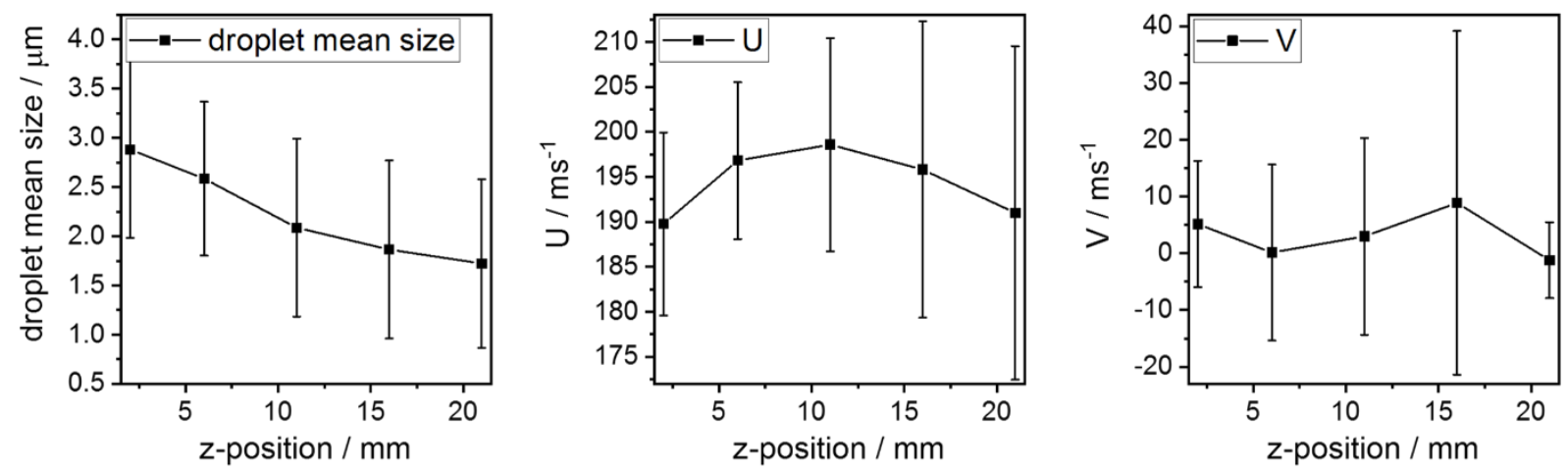

Figure 6. Left: Evolution of the droplet mean size as a function of the distance from the nozzle. Middle: Axial velocity $\mathrm{U}$ as a function as a function of the distance from the nozzle. Right: Axial velocity $\mathrm{U}$ as a function as a function of the distance from the nozzle. 


\section{S4. Estimation of RDX core diameters and TNT shell thicknesses for isotropic hexolite core/shell nanoparticles}

The theoretical diameters of the RDX cores and the TNT shell thicknesses of the diverse isotropic hexolite core/shell nanoparticles were calculated from the RDX and TNT mass fractions $\left(\omega_{R D X}, \omega_{T N T}\right)$, the measured particle means radius $\left(r_{\text {hex }}\right)$ and the densities of RDX and TNT $\left(\rho_{R D X}, \rho_{T N T}\right)$. A typical isotropic core/shell particle is presented in Figure 7.

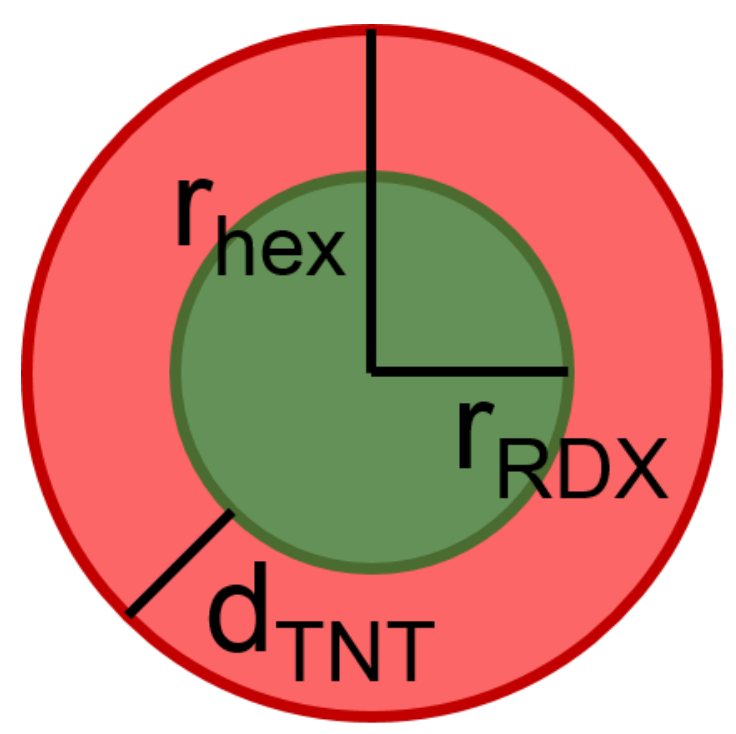

Figure 7. Schematic representation of an isotropic hexolite RDX/TNT core/shell nanoparticle; $r_{\text {hex }}$ gives the particle radius, $r_{R D X}$ represents the radius of the RDX core and $d_{T N T}$ the TNT shell thickness.

For a given RDX/TNT mass fraction ratio, the RDX and TNT masses of a hexolite core/shell nanoparticle behave like:

$\frac{m_{R D X}}{m_{T N T}}=\frac{\omega_{R D X}}{\omega_{T N T}}$.

Eq. 4

Furthermore, the densities of RDX and TNT behave like:

$\frac{\rho_{R D X}}{\rho_{T N T}}=\frac{\frac{m_{R D X}}{V_{R D X}}}{\frac{m_{T N T}}{V_{T N T}}}=\frac{m_{R D X}}{V_{R D X}} \cdot \frac{V_{T N T}}{m_{T N T}}$. 
The volume of a typical spherical hexolite core/shell nanoparticle can be given as the sum of RDX core volume and TNT shell volume:

$$
V_{\text {hex }}=V_{R D X}+V_{T N T}
$$

In order to calculate the mean radius $r_{R D X}$ of the RDX core, Eq. 4 is rearranged to $m_{T N T}$ and Eq. 5 is rearranged to $V_{T N T}$ :

$$
\begin{aligned}
& m_{T N T}=\frac{\omega_{T N T} \cdot m_{R D X}}{\omega_{R D X}} . \\
& V_{T N T}=\frac{m_{T N T} \cdot V_{R D X} \cdot \rho_{R D X}}{m_{R D X} \cdot \rho_{T N T}} .
\end{aligned}
$$

Inserting Eq. 7 in Eq. 8 results in:

$$
V_{T N T}=\frac{\omega_{T N T} \cdot V_{R D X} \cdot \rho_{R D X}}{\omega_{R D X} \cdot \rho_{T N T}}
$$

Inserting Eq. 9 in Eq. 6 and rearranging towards $V_{R D X}$ result in:

$$
V_{R D X}=\frac{V_{h e x}}{1+\frac{\omega_{T N T}}{\omega_{R D X}} \cdot \frac{\rho_{R D X}}{\rho_{T N T}}}
$$

By using the sphere volume $\left(V=\frac{4}{3} r^{3} \pi\right), r_{R D X}$ can be finally given as:

$$
r_{R D X}=\frac{r_{\text {hex }}}{\sqrt[3]{1+\frac{\omega_{T N T}}{\omega_{R D X}} \cdot \frac{\rho_{R D X}}{\rho_{T N T}}}} .
$$

Finally, the mean diameter of the TNT shell thickness is: 
Calculated RDX core radii and diameters for the diverse assumed isotropic hexolite RDX/TNT core/shell nanoparticles are summed up in Table 3. The density of RDX amounts $1.82 \mathrm{~g} / \mathrm{cm}^{3}$, the density of TNT is given with $1.65 \mathrm{~g} / \mathrm{cm}^{3}$.

Table 3. Estimated RDX core radii and diameters and TNT shell thicknesses for isotropic hexolite core/shell nanoparticles

\begin{tabular}{|r|r|r|r|r|r|}
\hline $\begin{array}{c}\text { mass fraction } \\
\text { ratio } \\
\text { (RDX/TNT) }\end{array}$ & \multicolumn{1}{c|}{$\begin{array}{c}\text { particle } \\
\text { mean size }\end{array}$} & \multicolumn{1}{c|}{$\boldsymbol{r}_{\boldsymbol{h e x}}$} & \multicolumn{1}{c|}{$\boldsymbol{r}_{\boldsymbol{R} \boldsymbol{X}}$} & \multicolumn{1}{c|}{$\begin{array}{c}\text { RDX core } \\
\text { diameter }\end{array}$} & $\begin{array}{c}\text { TNT shell } \\
\text { thickness }\end{array}$ \\
\hline $60 / 40$ & $32.2 \mathrm{~nm}$ & $16.1 \mathrm{~nm}$ & $13.4 \mathrm{~nm}$ & $26.7 \mathrm{~nm}$ & $2.7 \mathrm{~nm}$ \\
\hline $40 / 60$ & $29.4 \mathrm{~nm}$ & $14.7 \mathrm{~nm}$ & $10.6 \mathrm{~nm}$ & $21.2 \mathrm{~nm}$ & $4.1 \mathrm{~nm}$ \\
\hline $20 / 80$ & $59.7 \mathrm{~nm}$ & $29.9 \mathrm{~nm}$ & $17.0 \mathrm{~nm}$ & $34.0 \mathrm{~nm}$ & $12.9 \mathrm{~nm}$ \\
\hline
\end{tabular}

\section{References}

1. Dreger, Z. A.; Gupta, Y. M., High Pressure Raman Spectroscopy of Single Crystals of Hexahydro-1,3,5-trinitro-1,3,5-triazine (RDX). The Journal of Physical Chemistry B 2007, 111, 3893-3903.

2. Miao, M. S.; Dreger, Z. A.; Winey, J. M.; Gupta, Y. M., Density Functional Theory Calculations of Pressure Effects on the Vibrational Structure of $\alpha$-RDX. The Journal of Physical Chemistry A 2008, 112, 12228-12234.

3. Deckert-Gaudig, T.; Pichot, V.; Spitzer, D.; Deckert, V., High-Resolution Raman Spectroscopy for the Nanostructural Characterization of Explosive Nanodiamond Precursors. ChemPhysChem 2017, 18, 175-178.

4. Clarkson, J.; Smith, W. E.; Batchelder, D. N.; Smith, D. A.; Coats, A. M., A Theoretical Study of the Structure and Vibrations of 2, 4, 6-Trinitrotolune. J. Mol. Struct. 2003, 648, 203214. 
Supporting Informnation - Formation Mechanism of Anis... (795.94 KiB) view on ChemRxiv • download file 